\title{
JOURNAL OF GUIDANCE, CONTROL, AND DYNAMICS
}

VOLUME 24, NUMBER 3

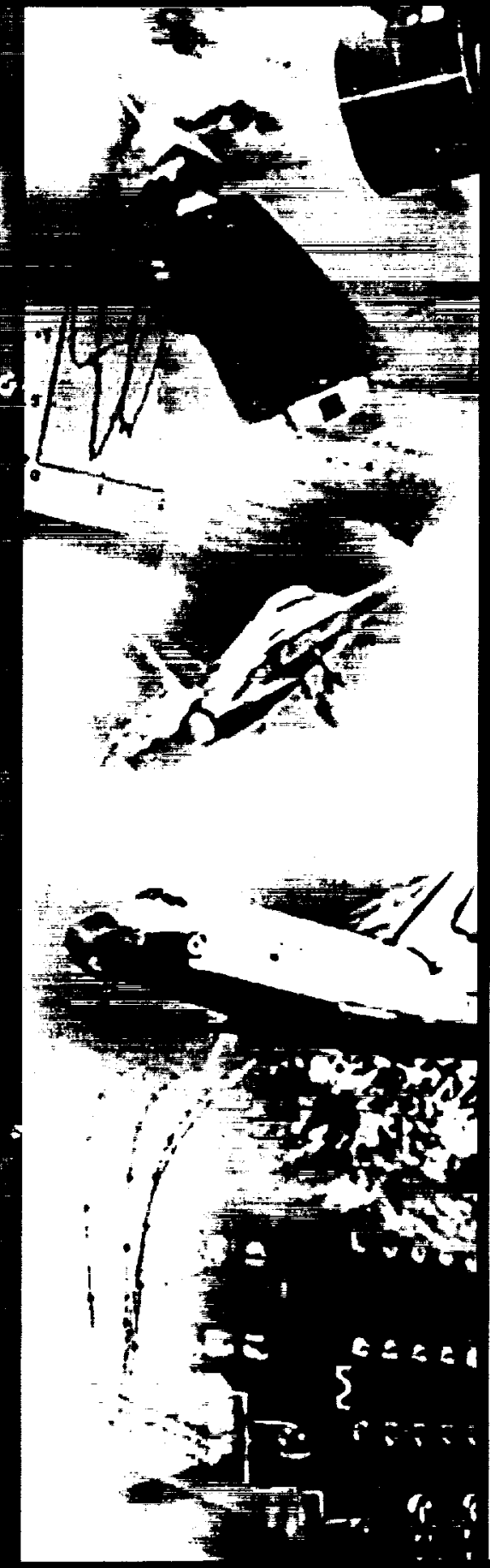

MAY-JUNE 2001

\section{FULL-LENGTH PAPEFS}

Vibration Reduction for Flexible Spacecraft Following Momentum Dumping

WithiWithout Slewing .............. . K. Banerjee, N. Pedreiro, W. E. Singhose

Markov Model of Terrair for Evaluation of Ground Proximity Warning System Thresholds J. K. Kuchar

Angular-Rate Estimation Using Delayed Quaternion Measurements

R. Azor, I. Y. Bar-Itzhack, J. K. Deutschmann, R. R. Harman

Methodology for the Performance Evaluation of a Conflict Probe . . . . K. D. Bilimoria Neoclassical Guidance for Homing Missiles ... . P. Gurfil, M. Jodorkovsky, M. Guelman State-Space Interpolation for a Gain-Scheduled Autopilot . . . . . . . . . D. J. Stilwell

Dynamics-Based Attitude Determination Using the Global Positioning System

C. Chun and F. C. Park

Attitude Sensing Using a Global-Positioning-System Antenna on a Turntable

$\ldots \ldots \ldots \ldots \ldots \ldots \ldots \ldots \ldots \ldots \ldots \ldots \ldots \ldots \ldots \ldots \ldots \ldots \ldots .$. . Psiaki

Two Reconfigurable Flight-Control Design Methods: Robust Servomechanism and

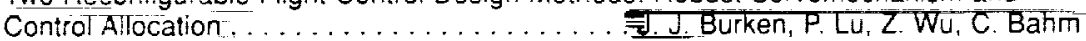

Control of Linear Second-Order'Systems by Fuzzy Logic-Based Algorithm

K. Cohen, T. Weller, J. Z. Ben Asher

Adaptive Output Feedback Control of an Aeroelastic System with Unstructured

Uncertainties .......................... R. Zhang and S. N. Singh

Application of Control Structure Design Methods to a Jet Engine ........ Härefors Computationally Efficient Control Allocation.

W. C. Durham

Frequency-Domain Estimation of Parameters from Flight Data Using Neural Networks A. K. Ghosh and S. C. Raisinghani

Category II Pilot in-the-Loop Oscillations Analysis from Robust Stability Methods

F. Amato, R. lervolino, S. Scala, L. Verde

Modeling and Simulation of Flexible Flight Vehicles ... . D. K. Schmidt and D. L. Raney

Improved Deployment Characteristics of a Tether-Connected Munition System

G. Frost and M. Costello

Mixed Norm Control of a Helicopter

M. Trentini and J. K. Pieper

Aircraft Nonminimum Phase Control in Dynamic Sliding Manifolds

$\ldots \ldots \ldots \ldots \ldots \ldots \ldots \ldots \ldots \ldots$. A. Shkolnikov and Y. B. Shtessel

Influence of Unstable Manifolds on Orbit Uncertainty ... D. J. Scheeres, D. Han, Y. Hou

566

573

Table of Contents continued on back cover

\section{C.TH:}

A Publication of the American Institute of Aeronautics and Astronautics Devoted to the Technology of Dynamics and Control JGCODS 24(3) 417-640 (2001)

ISSN 0731-5090 


\title{
Two Reconfigurable Flight-Control Design Methods: Robust Servomechanism and Control Allocation
}

\author{
John J. Burken* \\ NASA Dryden Flight Research Center, Edvards, Culifornia 93523 \\ Ping $\mathrm{Lu}^{\dagger}$ and Zhenglu $\mathrm{Wu}^{\ddagger}$ \\ Iowa State University, Ames, Iow'a 500II-227I \\ and \\ Cathy Bahm* \\ NASA Dryden Flight Research Center. Edwards, California 93523
}

\begin{abstract}
Two methods are discussed for design of reconfigurable flight-control systems when one or more control surfaces are jammed. The first is a robust servomechanism control approach, which is a generalization of the classical proportional-plus-integral control to multi-input/multi-output systems. The second proposed method is a controlallocation approach based on a quadratic programming formulation. The formulation is formally analyzed, and a globally convergent fixed-point iteration algorithm is used to make onboard implementation of this method feasible. The two methods are applied to reconfigurable entry flight control design for the X-33 vehicle. Nonlinear six-degree-of-freedom simulations demonstrate simultaneous tracking of angle-of-attack and roll-angle commands during control surface failures. The control-allocation method appears to offer more uniform and good performance at the expense of modestly higher computation requirement.
\end{abstract}

\section{Introduction}

$\mathbf{F}$ LIGHT-CONTROL system reconfiguration is concerned with making changes in fight-control system to adapt to failures and damages. The adaptation is usually in the forms of control system gain changes or control law changes. Reconfigurable control offers the potential of significant enhancement of flight safety and mission success rate. The reconfigurable controller has the potential of landing a crippled airplane safely and thus increasing mission success rates. Because of its clear benefits in both military and civil applications, flight-control reconfiguration research has received considerable attention in recent years, exemplified by the U.S. Air Force Reconfigurable Control for Tailless Fighter Aircraft program' and the NASA X-33 program.' The references contained in Ref. I provide a glimpse of some previous studies on this subject. An often investigated approach for reconfigurable control system design is through some form of model-following technique, typically by means of either parameter adaptation ${ }^{3}$ or neural networks. ${ }^{1}$ More recently, reconfigurable control designs using dynamic inversion and neural network to adaptively cancel inversion errors have demonstrated good performance. ${ }^{4}$

A major task in control reconfiguration deals with adjusting the controller gains on-line or switching to a different control law to compensate for the failure. This paper focuses on this aspect. More specifically, the failure is assumed to be a jammed aerodynamic effector. which is among class 1 failures as defined in Ref. I. It will be further assumed, when necessary, that the aircraft is fitted with smart actuators so that the jammed control surfaces can be detected and the position identified. This condition is already readily met by actuators available today.

The objective of this work is to seek reconfigurable control system designs that are easily implementable in flight sof ware, reliable,

Received 22 April 1999; revision received 10 November 2000; accepted for publication 30 November 2000 . Copyright (C) 2001 by the American Institute of Aeronautics and Astronautics. Inc. No copyright is asserted in the United States under Title 17. U.S. Code. The U.S. Government has a royaltyfree license to exercise all rights under the copyright claimed herein for Govemmental purposes. All other rights are reserved by the copyright owner.

- Aerospate Engineer. Member AIAA.

-Associate Professor, Department of Aerospace Engineering and Engineering Mechanics: plu@ iastate.edu. Associate Fellow AIAA.

$\ddagger$ Griduate Student, Department of Atrospace Enginesring and Engineering Mechanics. and offer a degree of assurance of success for the targeted types of failures. The reconfigured control system is expected to restabilize the aircraft should control surface jamming occur and provide reasonable command-tracking performance.

To these ends, two approaches are investigated and evaluated in this paper. One is based on the robust servomechanism design, ${ }^{3 .}$ which is a generalization of the classical proportional-plus-integral (PI) design. In this approach the effect of the jammed surface is treated as a disturbance to the system. A PI controller is designed to stabilize the aircraft (stabilization), balance the jammed surface (disturbance rejection), and provide command tracking. The second method is developed by the authors of this paper, which is based on a control-allocation approach. Here a reference baseline control law is ficst designed for the healthy aircraft with all of the control surface operable. At any given state the desired control inputs (e.g., moments and forces) to the aircraft can be computed from this baseline control law. In the event of a jammed surface, the redundant degree of freedom of the control effectors is utilized to distribute the deflections of the operable surfaces in an optimal fashion so as to cancel the influence of the jammed surface and reproduce as closely as possible the desired inputs the reference controller would have produced to the aircraft.

As a demonstration, the two approaches are applied to a reconfigurable entry fight-control system design for the $X-33$ vehicle. The $\mathrm{X}-33$ vehicle is a one-half-scale suborbital protolype single-stageto-orbit reusable launch vehicle. In flight tests the $X-33$ vehicle will accelerate 10 approximately Mach 9 and climb to an altitude of approximately $200,000 \mathrm{ft}$. The entry flight immediately follows a shor transition phase after the ascent. Figure 1 shows the configuration of the X-33. The X-33 has four sets of control surfaces: rudders. body flaps, inboard and outboard elevons, with left and right side for each set. Each of the eight surfaces can be independently actuated with one actuator for each surface. All of the aerosurfaces will use electromechanical actuators (EMAs). The body flaps also have an pneumatic load assist device that can be used for a total of $40 \mathrm{~s}$ during ascent or entry.

Analysis has shown that although the probability for an actuator failure is very low. when it does happen, the failure would most likely result in jam of the associated aerosurface. 2 The EMAs are capable of failure detection and identification within 120-160 ms. This capability provides information on which surface, if any, is jammed, and jammed at what position. The reconfigurable control design is 


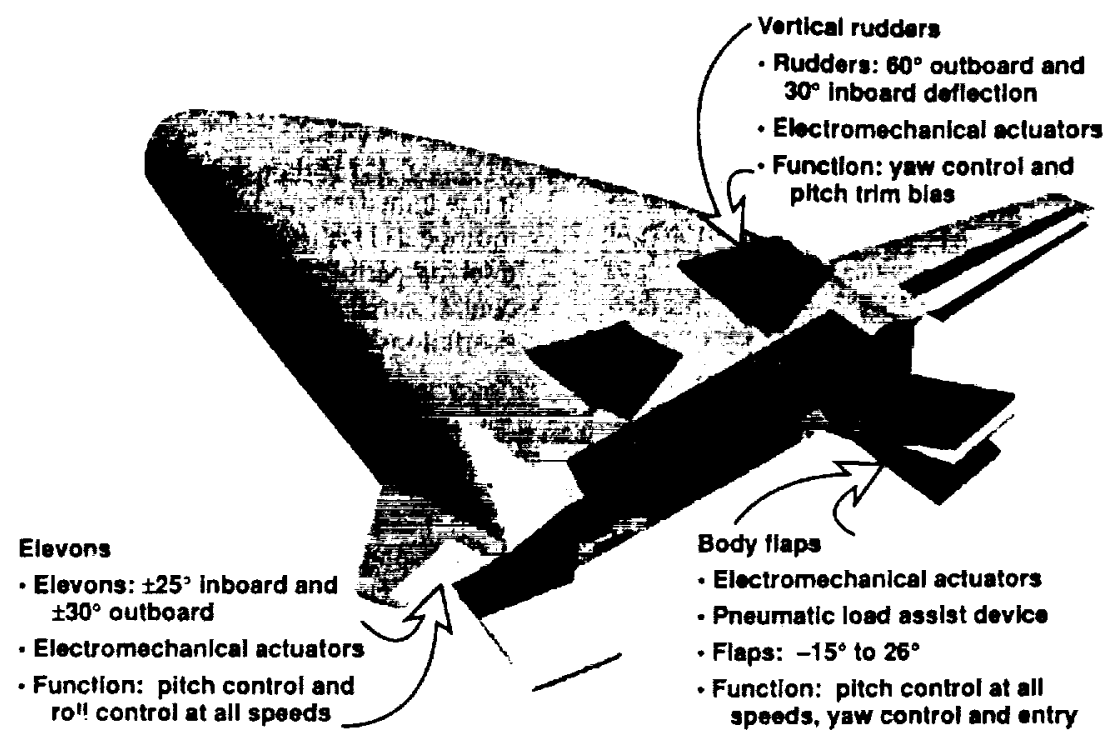

Fig. $1 \quad \mathrm{X}-33$ advanced technology demonstrator.

done for each surface failure. The eight control surfaces have control power capable of providing redundant pitch, roll, and yaw restoring moments such that if one surface fails, the potential exists for an alternate control scheme to maintain control of the vehicle.

The control system reconfiguration problem for the $X-33$ is posed as follows: If a single control surface fails (jams, floats, or runs away). can the nominal or reconfigurable controller be used to land the vehicle safely? A nominal controller has some inherent robustness and may be able to handle a limited failure set (such as a left rudder jammed at $3 \mathrm{deg}$ ). An appropriately designed reconfizurable controller should have a much larger region of survivable failure conditions. The two proposed reconfigurable control designs appear to meet this challenge well.

The methodology for the robust servomechanism design is reviewed in Sec. II. In Sec. III, the control-allocation method is introduced as a quadratic programming (QP) problem: two analytical yet practically useful properties of this formulation are established: and a globally convergent fixed-point iteration algorithm suitable for onboard implementation is described. Section IV contains the application of the two methods to reconfigurable entry flight control of the $\mathrm{X}-33$ vehicle and the evaluation of the performance. Conclusions are given in Sec. V.

\section{Servomechanism Problem for Multi-Input/Multi-Output Systems}

The servomechanism problem is concerned with control of a dynamic system to achieve asymptotic tracking of desired output and rejection of unmeasurable disturbance. For single-input/singleoutput (SISO) systems the problem has been well understood for 50 years. However, it is only in the last two decades that this problem has been solved for multi-input/multi-output (MIMO) systems. In the following a simpler version of the problem is introduced, and the controller design methodology is reviewed. For more complete discussion and detail the reader is referred to Refs. 5 and 6.

\section{A. Problem Formulation}

Consider a linear, time-invariant, MIMO system:

$$
\begin{aligned}
& \dot{x}=A x+B u+E u \\
& y=C x+D u+F u
\end{aligned}
$$

where $x \in R^{\prime \prime}$ is the plant state, $u \in R^{m}$ the plant input. $u \in R^{\prime}$ the disturbance, and $y \in R^{n}$ the controlled output with $p \leq m$. Let $r \in R^{n}$ represent the desired history for $\because$. Assume further that $r$ and $u$ are modeled by

$$
\begin{gathered}
r^{(q)}+\alpha_{1} r^{(y-11}+\cdots+\alpha_{q-1} \dot{r}+\alpha_{q} r=0 \\
w^{(q)}+\alpha_{1} w^{(q-1)}+\cdots+\alpha_{q-1} \dot{w}+\alpha_{q} w=0
\end{gathered}
$$

with $q \geq 1$. The preceding formulation encompasses many commonly used signals. including constants (when $q=1$ and $\alpha_{1}=0$ ), polynomials (when $\alpha_{i}=0, i=1, \ldots q$ ), sinusoidal functions (when $q=2, \alpha_{1}=0$, and $\alpha_{2}>0$ ) and exponential functions. The initial conditions for $u$ are assumed to be arbitrary; therefore, $u(t)$ is considered unknown (unmeasurable). In a more general formulation the dynamics of $r(t)$ and $u^{\prime}(t)$ do not have to be the same. ${ }^{6}$ But they are assumed to be the same here for simplicity, and such an assumption is adequate for our specific purpose in this paper. The objectives of the control design are to find a feedback controller such that 1) the closed-loop system is stable: 2$)$ the error $e(t)=r(t)-y(t)$ approaches zero in the presence of the unmeasurable (possibly timevarying) disturbance $u(t)$; and 3 ) the closed-loop system is robust in the sense that asymptotic tracking of $r$ and rejection of $u$ are maintained in the presence of system parametric uncertainty or even variations in the order of the dynamics, as long as the closed-loop system remains stahle.

\section{B. Robust Servomechanism Design Methodology}

A dynamic controller is to be designed to meet the objectives just stated. The controller dynamics are set to be

$$
\dot{x_{c}}=A_{1} \cdot x_{c}+B_{c}(r-y)
$$

where $x_{a} \in R^{p y}$ is the controller state and $A_{c} \in R^{p q \times p q}=$ block diag $[\Gamma \ldots . . \Gamma]$ with

$$
\Gamma=\left[\begin{array}{ccccc}
0 & 1 & 0 & \cdots & 0 \\
0 & 0 & 1 & \cdots & 0 \\
\vdots & \vdots & \vdots & \vdots & \vdots \\
0 & 0 & \cdots & 0 & 1 \\
-\alpha_{1} & -\alpha_{1,-1} & \cdots & -\alpha_{2} & -\alpha_{1}
\end{array}\right] \in R^{q \times q}
$$

and $B_{1} \in R^{p q^{\times n}}=$ block diag $[\gamma, \ldots \gamma \mid$ with

$$
\gamma=\left[\begin{array}{c}
0 \\
\vdots \\
0 \\
1
\end{array}\right] \in R^{\prime \prime}
$$


Consider the open-loop system including the plant [Eq. (1)] and the controller dynamics [Eq. (5)] with $r=0$

$$
\left[\begin{array}{l}
\dot{x} \\
\dot{x}_{r}
\end{array}\right]=\left[\begin{array}{cc}
A & 0 \\
-B_{1} C & A_{c}
\end{array}\right]\left[\begin{array}{l}
x \\
x_{r}
\end{array}\right]+\left[\begin{array}{c}
B \\
-B_{c} D
\end{array}\right] u
$$

Let $\lambda_{1}, \ldots, \lambda_{4}$ be the roots of the polynomial

$$
\lambda^{4}+\alpha_{1} \lambda^{4-1}+\cdots+\alpha_{i t} \lambda \lambda+\alpha_{i q}=0
$$

Suppose that the following condition is satisfied:

$$
\operatorname{rank}\left[\begin{array}{cc}
\lambda_{1} l-A & B \\
-C & D
\end{array}\right]=n+p, \quad i=1, \ldots, q
$$

then the augmented system (8) is controllable ${ }^{5.6}$ Hence there exist control laws

$$
u=k x+k_{r} \cdot x_{i}
$$

such that the closed-loop system is stable. Furthermore, when $r \neq 0$ and satisfies Eq. (3), asymptotic tracking and disturbance rejection are achieved by such a control law. That is, $e=r-y \rightarrow 0$ for any initial condition $x(0)$ and any $u$ satisfying Eq. (4). The closed-loop system possesses robustness in the sense that for any (not necessarily small) perturbations in $\left\{A, B, C, D, B_{6}, k, k_{c}\right\}$, asymptotic tracking and disturbance rejection still hold as long as the closed-loop system

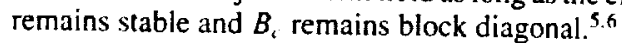

Remarks:

I) Because the augmented system (8) is controllable, the control law (10) can be conveniently found by applying the linear quadratic regulator (LQR) approach to $\mathrm{Eq}$. (8).

2) In the special case where $r$ is constant commandand $w$ is constant (but possibly unknown) disturbance, we have $q=\mathrm{I}$ and $\alpha_{1}=0$; therefore, $A_{c}=0$, and $B_{c}=I_{p \times p}$ according to their definitions. From the controller dynamics (5) it can be seen that $x_{i}=\int(r-y) \mathrm{d} t=\int e \mathrm{~d} t$. Thus the control law (10) is simply a PI control law, which is well known in classical SISO control theory. But the current formulation is much more general in that it applies to MIMO systems and allows tracking of time-varying commands and rejection of time-varying disturbances.

3) The principle of this class of methods is also alternatively known as the internal model principle in the literature because the controller has to include the models of the disturbance and command in order to achieve disturbance rejection and command tracking.

4) This robust servomechanism design applies conveniently to control of impaired aircraft with one or more jammed control surfaces. Suppose that the dynamic model (1) represents the linearized dynamics of such an aircraft at a trim condition. Let $w$ in Eq. (1) denote the (constant) position of the jammed surface and $u$ all of the remaining operable surfaces. The exact value of $w$ does not need to be known with this method. The matrix $E$ (a column vector in this case) in Eq. (1) is then the stability derivative associated with the surface now jammed (i.e., the column corresponding to the jammed surface in the $B$ matrix of the linearized model prior to failure). Now the problem is cast into the formulation in Sec. II.A anda PI controller (10) can be designed by LQR or pole-placement methods to stabilize the aircraft, reject the influence of the jammed surface, and track constant commands.

\section{Control Reconfiguration via Control Allocation}

Control allocation is concerned with how to distribute the deflections of multiple control surfaces of the aircraft to generate required control inputs (pitch, roll, and yaw moments, and forces) when the number of the control surfaces is greater than the number of required control inputs. Reference 7 contains a list of recent work on control allocation and provides exploratory discussions to several control allocation approaches based on quadratic and linear programming. In this section we consider a control reconfiguration approach, also based on a control allocation scheme using the QP method. The idea is to use the redundancy of the operable control surfaces to cancel the effects of the jammed surface and still provide the same (or almost the same) desired control inputs. Clearly the greater the control redundancy is, the better suited this approach would be. Also, this approach will require the position of the jammed surface to be known. either through the use of smart actuator or by estimation.

\section{A. Problem Formulation}

Let the linearized dynamics of the normal aircraft at a trim condition be given by

$$
\dot{x}=A x+B u
$$

It is assumed that a reference control law has been designed based on the model (11) that provides satisfactory stabilization and commandtracking performance for the aircraft. Suppose now that one of the control surfaces is suddenly jammed at a position $u$. Rewrite the postfailure state equation of the system (11) as

$$
\dot{x}=A x+B_{r} u_{r}+d_{x}
$$

where $u_{r} \in R^{m}$ represents the remaining control surfaces, $B_{r}$ the postfailure control influence matrix. $d_{x}=b_{w} w$ is the input to the aircraft caused by the jammed surface $w$, where $b_{y}$ is the column in $B$ corresponding to the jammed surface. Note that $d_{1}$ is known when $w$ is known. Let $z=C_{z} x$ be a selected $i$-dimensional controlled output vector $\left(z \in R^{i}\right)$ to be used in defining the control allocation. From Eq. (12) and the definition of : we have

$$
z=C_{z} A x+C_{z} B_{r} u_{r}+C_{z} d_{x} \triangleq A_{z} \cdot x+B_{z} u_{r}+d_{z}
$$

The choice of $z$ is not necessarily the same as $y$ in Sec. II, but must be one such that the closed-loop stability and performance can be ensured from control of $z$. From a control theoretical viewpoint the closed-loop stability requirement implies that the choice of the controlled output $z$ should lead to a nonminimum-phase system. One most natural choice of $z$ is $z=(p r q)^{T}$ (the roll, yaw, and pitch rates). Other choices are also possible. We require in general that $m \geq i$ in this approach (i.e, the number of operable control surfaces is greater than that of the controlled variables). At the current state $x(t)$ suppose that the reference baseline feedback control law for the healthy aircraft would have produced input $u^{*} \in R^{m+1}$ if all of the control surfaces were operable. Then the desired rate of $z$ would be

$$
\Sigma^{*}=C_{z} A x+C_{i} B u^{*}
$$

We seek a $u_{r}$ that makes the right-hand side of Eq. (13) as close as possible to that of Eq. (14), or $B_{z} u_{r}+d_{z} \approx C_{z} B u^{*}$. Thus the actual $\dot{z} \approx z^{*} ;$ consequently, $z$ remains close to $z^{*}$, which represents desired performance. Such a $u_{r}$ can be determined by minimization of the following quadratic function:

$$
\begin{aligned}
\min _{u_{r}} J & =\frac{1}{2}\left[( 1 - \varepsilon ) ( B _ { : } u _ { r } + d _ { z } - C _ { z } B u ^ { * } ) ^ { T } Q _ { 1 } \left(B_{:} u_{r}+d_{z}\right.\right. \\
& \left.\left.-C_{z} B u^{*}\right)+\varepsilon u_{r}^{T} Q_{2} u_{r}\right]
\end{aligned}
$$

for some small $0<\varepsilon<1$ and subject to

$$
u_{r \min } \leq u_{r} \leq u_{r \max }
$$

where $Q_{1}$ and $Q_{2}$ are positive definite matrices of appropriate dimensions. The $u_{r \min }$ and $u_{r \max }$ in $\mathrm{Eq} .(16)$ are the lower and upper bounds of the remaining control surfaces.

The minimization of $J$ subject to Eq. (16) constitutes a QP problem. The term $\varepsilon u_{r}^{T} Q_{2} u_{r}$ in Eq. (15) is a regularization term to the QP problem. Without it $(\varepsilon=0)$, the Hessian of $J$, $\partial^{2} J / \partial u_{r}^{2}=B_{T}^{T} Q_{1} B_{z} \in R^{m \times m}$, is not strictly positive definite because the rank of $B_{:}^{T} Q_{1} B_{z} \in R^{m \times m}$ is at most $i$ but $m>i$. In this case the QP problem has no unique solution, and chattering in $u_{r}$ can easily occur. On the other hand, any $0<\varepsilon<1$ will make the Hessian of $J$ positive definite, and the solution to the QP problem is unique. But clearly $\varepsilon$ should be sufficiently small in order for $B_{z} u_{r}+d_{z}-C_{z} B_{u^{*}} \approx 0$. When $B_{z} u_{r}+d_{z}-C_{z} B u^{*} \approx 0$, the response of the aircraft would be very close to that of the healthy aircraft, despite the jamming of a control surface.

\section{B. Analysis}

To formalize the preceding heuristic arguments about the rationale of the formulation for control allocation, let us define for given
$B_{-}, d_{-}$, and $C_{-} B \|^{*}$ the set

$$
\mathcal{U}=\left\{u_{r} \in R^{m} \mid B_{z} u_{r}+d_{z}-C_{z} B u^{*}=0, \quad u_{r \min } \leq u_{r} \leq u_{r \max }\right\}
$$


Denote the solution to the QP problem (15) and (16) by $u_{r}^{*}(\varepsilon)$ for a given $0 \leq \varepsilon<1$. We lirst make the following statement:

\section{Property 1}

If $\mathcal{U}$ is not emply, the solution $u_{r}^{*}$ of the QP problem (15) and (16) has the asymptotic property of

$$
\lim _{x \rightarrow 0}\left[B: u_{r}^{*}(\varepsilon)+d_{z}-C_{z} B u^{*}\right]=0
$$

When $\varepsilon=0$, any $u$, in $\mathcal{U}$ can be taken to be $u_{r}^{*}$, and $J\left(u_{r}^{*}\right)=0$ is the minimum. For $\varepsilon \neq 0$ this claim can be proved by contradiction. Let $\delta_{\varepsilon}=B_{z} u_{r}^{*}(\varepsilon)+d_{z}-C_{z} B u^{*}$ for a given $\varepsilon$. Suppose that $\delta_{\varepsilon} \nrightarrow 0$ as $\varepsilon \rightarrow 0$. Take an arbitrary $\tilde{u}_{r} \in \mathcal{U}$. That is, $B \cdot \tilde{u}_{r}+d_{-}-C_{:} B u^{*}=0$, and $J\left(\tilde{u}_{r}\right)=\varepsilon \tilde{u}_{r}^{T} Q_{2} \tilde{u}_{r} / 2$. Then for sufficiently small $\varepsilon>0$, the following will be true:

$$
\begin{gathered}
J\left[u_{r}^{*}(\varepsilon)\right]=\frac{1}{2}\left[(1-\varepsilon) \delta_{\varepsilon}^{T} Q_{1} \delta_{\varepsilon}+\varepsilon u_{r}^{* T}(\varepsilon) Q_{2} u_{r}^{*}(\varepsilon)\right] \\
>\frac{1}{2} \varepsilon \tilde{u}_{r}^{T} Q_{2} \bar{u}_{r}=J\left(\tilde{u}_{r}\right)
\end{gathered}
$$

because $\delta_{\varepsilon}^{T} Q_{1} \delta_{\varepsilon} \nrightarrow 0$. But the result that $J\left[u_{r}^{*}(\varepsilon)\right]>J\left(\bar{u}_{r}\right)$ contradicts the assumption that $u_{r}^{*}$ is the solution of the QP problem. Therefore $\delta_{\varepsilon}=B_{z} u_{r}^{*}+d_{z}-C_{:} B u^{*}$ must approach zero as $\varepsilon \rightarrow 0$. In fact, from the requirement that $J\left(u_{r}^{*}\right)<J\left(\bar{u}_{r}\right)$, including the case of sufficiently small $\varepsilon>0$, we can further conclude that $\delta_{\varepsilon}$ must approach zero faster than $\sqrt{ } \varepsilon$ as $\varepsilon \rightarrow 0$ so that $\lim _{\varepsilon \rightarrow 0}\left[\delta_{\varepsilon}^{T} Q_{1} \delta_{\varepsilon} / \varepsilon\right] \rightarrow 0$.

This property formally establishes that we can achieve $B: u_{r}+$ $d_{z}-C_{z} B u^{*} \approx 0$ within any practically reasonable accuracy by solving the QP problem with a sufficiently small $\varepsilon>0$, whenever $B_{z} u_{r}+d_{z}-C_{z} B u^{*}=0$ has solutions within the bounds [Eq. (16)].

Our second claim applies to a more specific case. If rank $\left(B_{z}\right)=i$ (full rank), the equation $B_{z} u_{r}+d_{z}-C_{z} B u^{*}=0$ has infinitely many solutions $u_{r}$. But it is well known that the solution defined by the Moore-Penrose pseudoinverse

$$
u_{\min }^{*}=B_{z}^{T}\left(B_{z} B_{z}^{T}\right)^{-1}\left(C_{z} B u^{*}-d_{z}\right)
$$

is the unique one that has the minimum 2-norm $\|\cdot\|^{2}$ among all such $u_{r}$. We state that the solution of the preceding QP problem can be made as close to $\|_{\min }^{*}$ as desired.

\section{Propern 2}

If the matrix $B_{z}$ is of full rank and $u_{\min }^{*}$ defined in Eq. (20) satisfies the constraint $(1 \overline{6})$, then for $Q_{2}=I$ and sufficiently small $\varepsilon>0$ the QP problem (15) and (16) has the solution of

$$
u_{r}^{*}(\varepsilon)=u_{\min }^{*}+\eta_{\varepsilon}
$$

where $u_{\text {min }}^{*}$ is given in Eq. (20), $\eta_{\varepsilon} \in R^{m}$ and $\eta_{\varepsilon} \rightarrow 0$ as $\varepsilon \rightarrow 0$.

This property can be shown to be true as follows: The assumption that $u_{\min }^{*}$ satisfies Eq. (16) immediately leads to the conclusion of $u_{\min }^{*} \in \mathcal{U}$; thus, the set $\mathcal{U}$ is nonempty. By property $I$ the solution of the QP problem with small $\varepsilon$ can be expressed as $u_{r}^{*}(\varepsilon)=\tilde{u}_{r}+\eta_{\varepsilon}$ for some $\tilde{u}_{r} \in \mathcal{U}$ and $\eta_{\varepsilon} \in R^{m}$ with $\eta_{\varepsilon} \rightarrow 0$ as $\varepsilon \rightarrow 0$. When $Q_{2}=I$, the optimal objective function from Eq. (15) becomes

$$
\begin{aligned}
J\left(u_{r}^{*}\right) & =\frac{1}{2}\left[(1-\varepsilon) \delta_{\varepsilon}^{T} Q_{1} \delta_{\varepsilon}+\varepsilon u_{r}^{* T} u_{r}^{*}\right] \\
= & \frac{1}{2} \varepsilon\left[(1-\varepsilon)\left(\delta_{\varepsilon}^{T} Q_{1} \delta_{\varepsilon}\right) / \varepsilon+\left\|\tilde{u}_{r}\right\|^{2}+2 \eta_{\varepsilon}^{T} \bar{u}_{r}+\left\|\eta_{r}\right\|^{2}\right]
\end{aligned}
$$

where $\delta_{\varepsilon}$ is the same as defined in the discussion of property 1. From the observation in the discussion of property 1 that $\lim _{k \rightarrow 0}\left(\delta_{\varepsilon}^{T} Q_{1} \delta_{\varepsilon} / \varepsilon\right) \rightarrow 0$, clearly for a sufficiently small (but nonzero) $\varepsilon$, the dominant term in $J\left(u_{r}^{*}\right)$ is $\left\|\tilde{u}_{r}\right\|^{2}$ because all of the other terms in the brackets approach zero as $\varepsilon \rightarrow 0$. For $J\left(u_{r}^{*}\right)$ to be minimum, $\bar{u}_{r}$ must be the one in $\mathcal{U}$ with the minipum 2-norm. Hence $\tilde{u}_{r}=u_{\min }^{*}$. where $u_{\min }^{*}$ is given in Eq. (20).

Property 2 reveals that the QP formulation with $Q_{2}=I$ and a small $\varepsilon>0$ will produce a solution that is practically the same as the commonly employed pseudoinverse solution in control allocation, if such a solution satisfies the constraints (16). But allowing different choices of $Q_{2}$ in the QP formulation conveniently provides Hexibility to assign preference to small deflections of certain control surfaces, which is not easy to do if the control allocation is only based on enforcing $B_{z} u_{r}+d_{z}-C_{z} B u^{*}=0$.

The preceding discussion contirms formally that the QP formulation with a small $\varepsilon$ in $E_{4}$. (15) preserves the objective of redistributing $u_{r}$ to achieve $B_{z} u_{r}+d_{z}-C_{z} B u^{*} \approx 0$ whenever it is possible to do within the bounds [Eq. (16)]. When direct control allocation batsed on solving $B_{z} u_{r}+d_{z}-C_{z} B u^{*}=0$ subject to Eq. (16) is not possible (i.e., the set $\mathcal{U}$ is empty), the solution of the QP problem still provides a best possible $u_{r}$.

\section{Fixed-Point Iteration Algorithm}

A reliable, efficient, and simple algorithm is the key for this control-allocation approach to be practically useful. When none of the constraints in Eq. (16) are active, solving the QP problem is straightforward, and the solution $u_{r}$ is obtained from the unique solution of the linearalgebraic system $\partial J / \partial u_{r}=0$, which gives

$$
u_{r}^{*}=(1-\varepsilon)\left[(1-\varepsilon) B_{:}^{T} Q_{1} B_{i}+\varepsilon Q_{2}\right]^{-1} B_{:}^{T} Q_{1}\left(C_{i} B u^{*}-d_{i}\right)
$$

In more general cases wher some of the constraints in Eq. (16) are active, the standard QP aigorithms ${ }^{8}$ are more involved and not suited for onboard implementation and applications. But for the special class of QP problems such as in Eqs. (15) and (16) where there are only inequality constraints of the simple form [Eq. (16)], an extremely simple. globally convergent fixed-point iteration algorithm can be devised for onboard use. This method is described and used in Ref. 9 in a different context. Here we shall apply this method to the QP problem (15) and (16).

Define a vector saturator $s[\cdot]=\left(s_{1}[\cdot] \cdots s_{m 1}[\cdot]\right)^{T}: R^{m} \rightarrow R^{m}$ by

$$
s_{j}[\zeta]= \begin{cases}U_{j}, & \zeta_{j} \geq U_{j} \\ \zeta_{j}, & L_{j}<\zeta_{j}<U_{j}, \quad j=1,2, \ldots, m \\ L_{j}, & \zeta_{j} \leq L_{j}\end{cases}
$$

for any $\zeta=\left(\zeta_{1} \cdots \zeta_{m}\right)^{T} \in R^{m}$, where for the moment $U_{j}=u_{\max }$, and $L_{j}=u_{\min }$, equal to the upper and lower bound or the $j$ th component of $u_{r}$, respectively. Let $H=(1-\varepsilon) B_{:}^{T} Q_{1} B_{z}+\varepsilon Q_{2}$. Calculate the sfalar

$$
\omega=\left[\sum_{i=1}^{m} \sum_{j=1}^{m} h_{i j}^{2}\right]^{-\frac{1}{2}}
$$

where $h_{i j}$ are the elements of $H$. Then the solution to the QP problem (15) and (16) satisfies the following fixed-point equation:

$$
\begin{aligned}
u_{r}= & s\left[(1-\varepsilon) \omega B_{z}^{T} Q_{1}\left(C_{z} B u^{*}-d_{z}\right)\right. \\
& \left.-\left(\omega H-I_{m \times m}\right) u_{r}\right] \triangleq f\left(u_{r}\right)
\end{aligned}
$$

Furthermore, the fixed-point iteration

$$
u_{r}^{(k)}=f\left[u_{r}^{(k-1)}\right] . \quad k=1.2, \ldots, \quad \forall u_{r}^{(0)} \in R^{m}
$$

converges to the unique solution of the QP problem from any initial guess $u_{r}^{(0)}$.

The validity of this fixed-point algorithmis established by examining the necessary and sufficient conditions for the QP problem and using global contraction mapping theory. ${ }^{9}$ The unconstrained solution (23) is just a special case of Eq. (26) when none of the components of the saturator $s$ in Eq. (26) are active. In such a case $s[\zeta]=\zeta$; therefore, Eq. (26) simply reduces to Eq. (23). But it should be stressed that in general, the QP problem solution by $\mathrm{Eq}$. (26) is different from what is obtained by clipping off (saturating) the righthand side of Eq. (23).

The fixed-point iteration algorithm (27) is particularly suited for onboard implementation. If the initial guess $u_{r}^{(0)}$ is chosen to be the solution of the QP problem in the preceding control update cycle, the current $u_{r}$ should be obtained in just a few iterations from Eq. (27).

A similar constrained optimization problem was formulated in Ref. 2 for redistributing control surfaces after the failure. Although simulations showed good performance, the computation requirement using a standard optimization algorithm was deemed to be too 
inlensive for onboard implementation. Another concern was aboul the convergence ratte. These concerns appear to be satisfactorily ad dressed by the current algorithm.

Finally. this method ciul be easily molitied to accommodate both control surface amplitude and rate constraints. Suppose, in addition to the amplitude constraint (16) that the rate limit for the $j$ th surface is $i_{\text {max }}$, and the sampling time of the control system is $\Delta t$. The only moditications will be to redefine the bounds of the saturator |Eq. $(2+1)$ at each $/$ by

$$
\begin{aligned}
& U_{1}=\min \left\{u_{\text {max, }} \cdot \Delta r u_{\text {max }}+u_{1}(t-\Delta t)\right\} \\
& L_{1}=\max \left\{u_{\text {mmi, }} \cdot-\Delta r \dot{u}_{\text {max }}+u_{1}(t-\Delta t)\right\}
\end{aligned}
$$
where $u_{,}(t-\Delta l)$ is the calculated control command for $\|_{j}$ at the
earlier update.

\section{Reconfigurable Entry Flight-Control Designs for the $X \cdot \mathbf{3 3}$}

A brief description of the $X-33$ vehicle and its flight-control system has been given in the Introduction. The $X-33$ vehicle relies on engine thrust vectoring and aerosurfaces during the ascent phase. Duril. the entry phase, the $X-33$ will be controlled by aerosurfaces and reaction control jets. During the ascent phase, only marginal benefits of reconfiguration were shown because of the corrective forces of which the engine thrust vectoring is capable can overcome any failed surface position. This study presents the results for the entry phase because control reconfiguration has beei shown to have the greatest payoff during entry. The Appendix provides the linearized dynamic model at a critical entry condition of Mach 3.16. This operating point will be used to demonstrate the two design approaches introduced in the preceding sections because this flight condition is a critical. unstable lateral-directional point. Both the longitudinal and lateral-directional time histories will be shown because of coupling between axes following a surface failure.

Once the control laws were designed, they were implemented in a high-fidelity nonlinear six-degree-of-freedom simulator for the $X-33$. Unless indicated otherwise, all of the simulation results presented in this paper were from the nonlinear simulation.

\section{A. Determination of Trimmable Jam Positions}

Before proceeding with the reconfigurable control design, it is helpful to understand whether the vehicle can still be retrimmed with a particular acrosurface jammed at a given position. Rewrite the postfailure alircraft model as

$$
\dot{r}=A_{x}+B_{r} u_{r}+b_{s} \delta
$$

where as before $\delta$ is the jammed surfice position, $B_{r}$ is the postfailure $B$ matrix, $"$, the remaining control surfaces, and $b_{2}$ the sensitivity vector corresponding to the jammed surface. Let yien represent the three body angular (roll, yaw, and pitch) rates in the body frame. Suppose that $y_{\text {terl }}=C_{\text {ser. }}$. Clearly

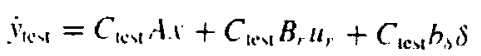

A necessary condition for retrimming the vehicle with the jammed surface is that the right-hand side of the preceding equation can still be made to vanish at $x=0$ with $a{ }^{\prime}$, in its allowable range. To tind the range of jammed positions of the surface $\delta$ for which retrim is possible, we may solve the following linear programming (LP) problem

$$
\min _{u_{r} . \delta} \delta \quad\left(\text { or } \max _{u_{f} . \delta} \delta\right)
$$

subject to

$$
\begin{gathered}
C_{\text {text }} B_{r} u_{r}+C_{\text {ter }} b_{j} \delta=0 \\
u_{r \min } \leq u_{r} \leq u_{r \max } . \quad \delta_{\min } \leq \delta \leq \delta_{\max }
\end{gathered}
$$

The solution of the LP problem (32-34) gives the minimum (most negative) or maximum jammed incremental position of $\delta$ that can be balanced at the trim condition by the remaining aerosurfaces $u$, within their deflection limits. This range found serves as a reasonable estimation of the limits within which the reconfigurable control system can still possibly stabilize the vehicle. In some cases including $\alpha, \beta$, and $v$ in $y_{\text {tex }}$ can give a more accurate estimate because the balance of forces is considered this way as well.

Applying this technique to the $X-33$ model in the Appendix, we found that for any jammed position within the physical limits of all aerosurfaces but the flaps the vehicle can always be retrimmed. For $\rho$ jammed body flap, however, it was found that $\delta_{\min }=-18.9$ (deg) and $\delta_{\max }=10$. (deg) (incremental from the trim position). This is because the body flaps are the predominant aerosurfaces for pitch control, and other aerosurfaces cannot adequately compensate for
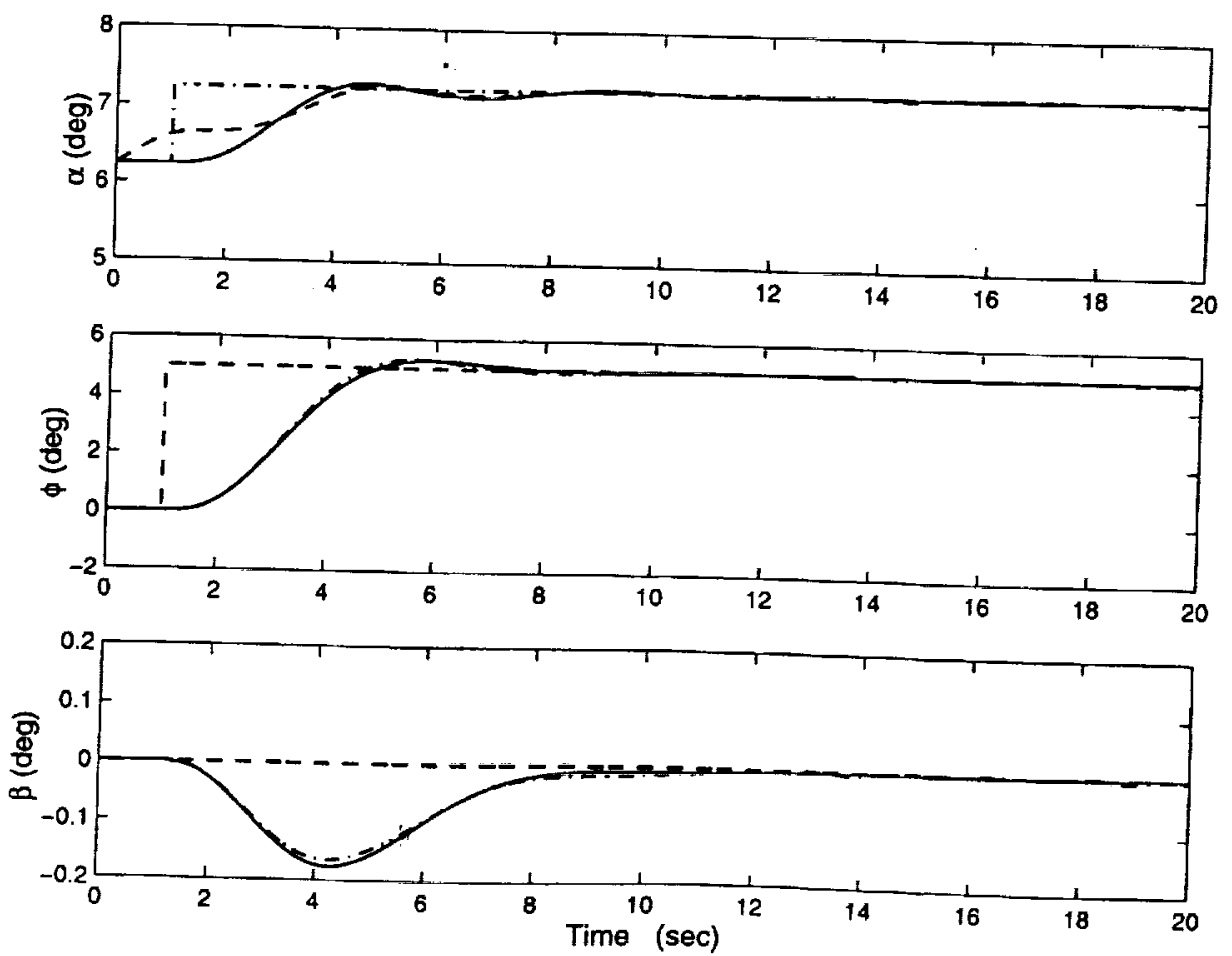

Fig. 2a $\mathrm{X}-3.3$ response from the linearized model (_) and nonlinear simulation (- - ) 
it if one of the llaps is jammed at a position far from their trim positions. When there are commands to be tracked, the range of recoverable body flap jammed positions will likely be even smaller than the range just found because tracking of the commands requires additional deflections of the remaining aerosurfaces. Also, because the preceding analysis is based on the linearized model, the actual trimmable limits based on the nonlinear model can be different to certain extent.

\section{B. Servomechanism Reconfigurable Design}

Following the method in Sec. II, we can design a PI-reconfigured control system for the jam of each surface. The gains of all eight such controllers will be stored onboard. Should the jam occur inflight, based on the information provided by the EMAs, an appropriate reconfigured controller will be switched on. The three outputs to be commanded are $\phi$ (roll angle), $\beta$ (sideslip angle), and $\alpha$ (angle of attack). The $\beta$ command is normally zero for coordinated flight. Although the linearized longitudinal and lateral directional dynamics are decoupled in the system matrix $A$. all of the control surfaces contribute to both longitudinal and lateral dynamics at different extents. Therefore the control design is carried out simultaneously for both directions. In design of the feedback PI control law, the forward velocity is ignored because it has negligible effect on the response. Assume constant commands $\phi_{\text {cmd }} . \beta_{\text {cmd. }}$, and $\alpha_{\text {smd. }}$. With
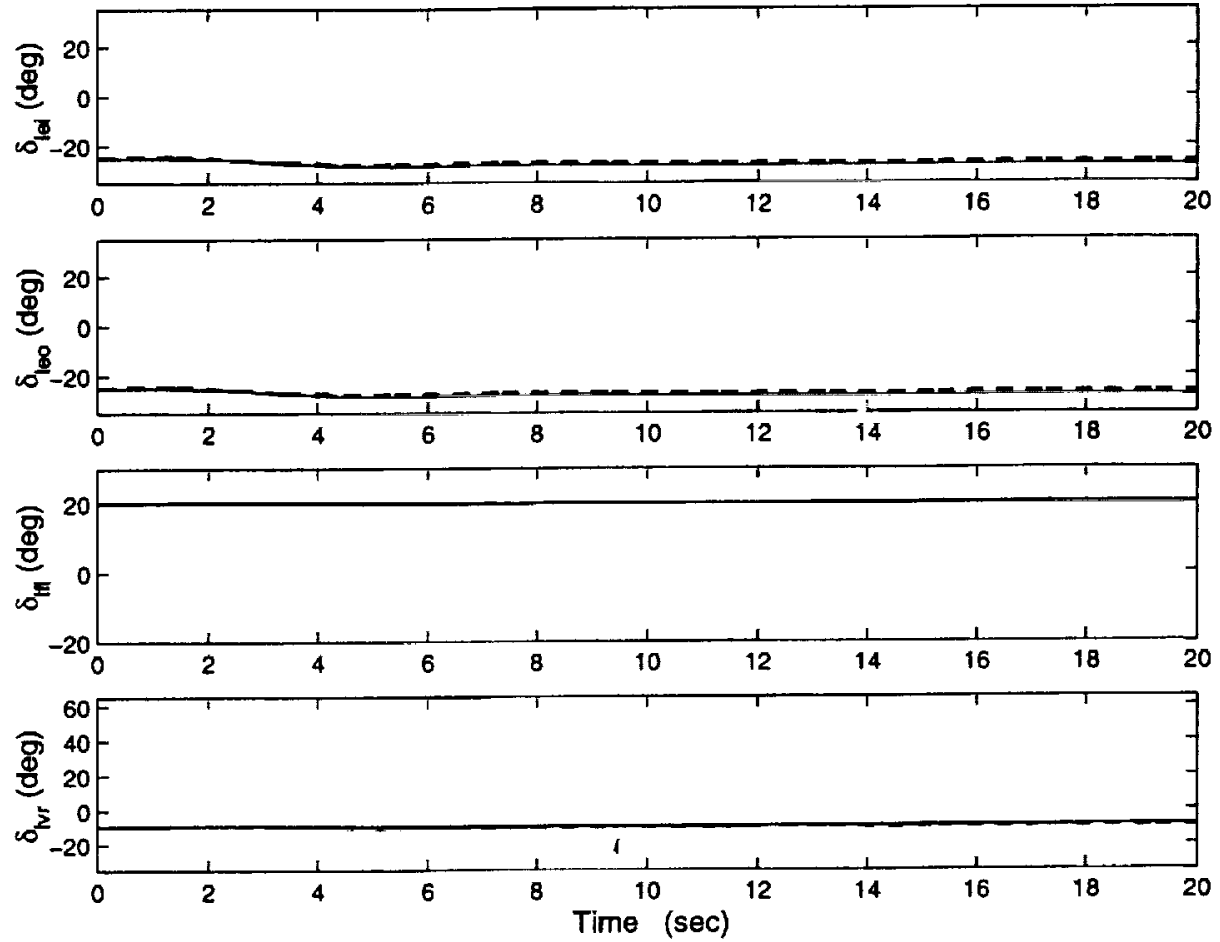

Fig. 2b Comparison of response from linearized model (-) and nonlinear model (---) for the left-side control surfaces.
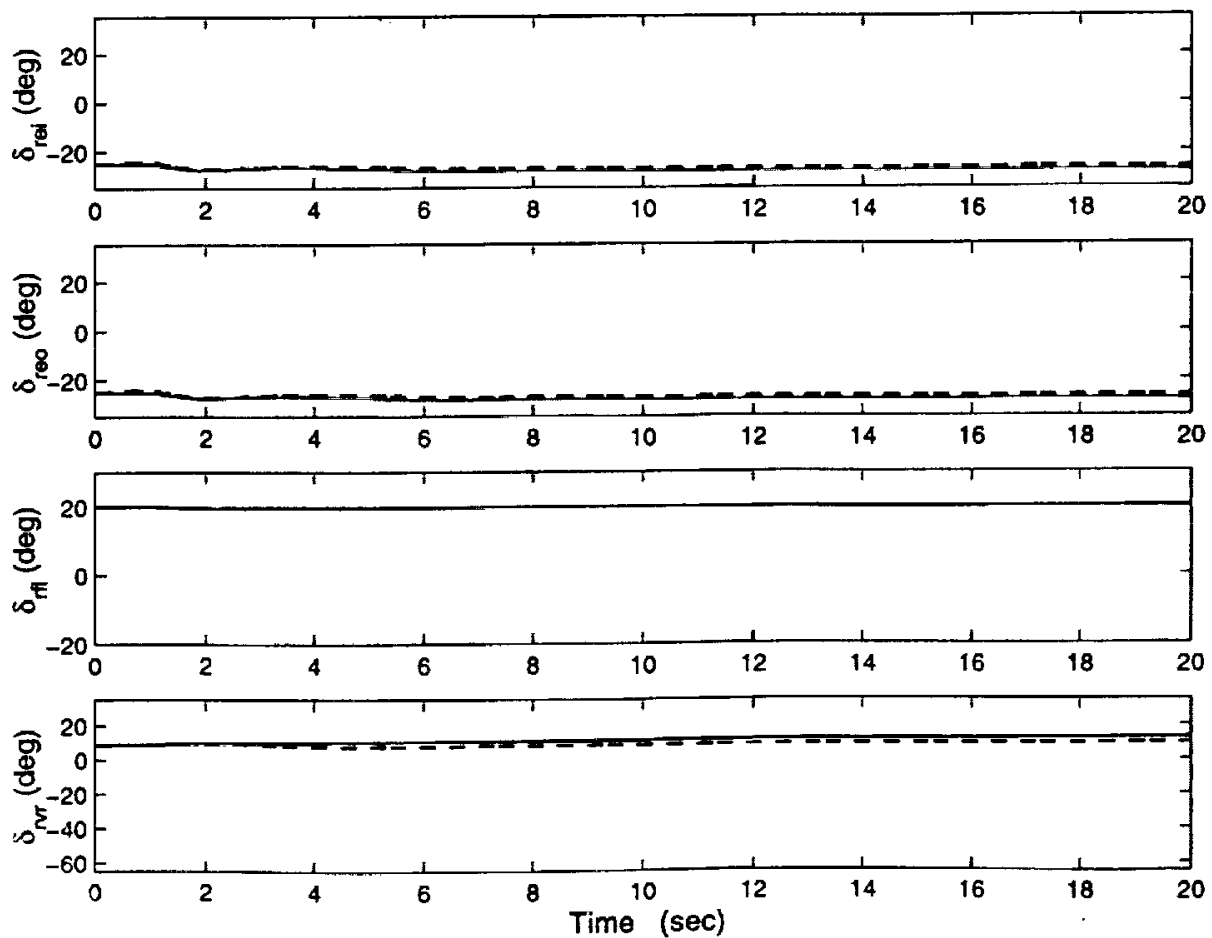

Fig. 2c Comparison of response from linearized model (- $\longrightarrow$ ) and nonlinear model (-- -) for right-side control surfaces. 

$y=(\phi H \alpha)^{r}$ and $r=\left(\phi_{\mathrm{cmd}} \beta_{\mathrm{cmu}} \alpha_{\mathrm{cmd}}\right)^{r}$, the controller dynamics
$(5)$ are now

$$
\dot{x}_{r^{1}}=\phi_{\mathrm{cmll}}-\phi, \quad \dot{r}_{r^{2}}=\beta_{\mathrm{cind}}-\beta, \quad \dot{x}_{i 3}=\alpha_{\mathrm{cmd}}-\alpha
$$

For each jammed surface the remaining seven surfaces and the eight vehicle states (excluding the forward velocity) plus the three integrators states [Eqs. (35)] constitute the augmented system (8). This augmented system is found to be controllable. An LQR control law for the augmented system, which is a PI control law for the X-33 in the form of

$$
\begin{aligned}
u_{r}= & K_{i} \cdot r+k_{\phi} \int\left(\phi_{\mathrm{cmd}}-\phi\right) \mathrm{d} t+k_{\phi} \int\left(\phi_{\mathrm{cmd}}-\beta\right) \mathrm{d} t \\
& +k_{\alpha} \int\left(\alpha_{\mathrm{cmdl}}-\alpha\right) \mathrm{d} t
\end{aligned}
$$

can be easily designed, where $x=(p r \beta \phi \psi \alpha q \theta)^{T}$. We have found that for each jammed surface, except for the flaps, a single set of gains are adequate to handle any jammed position within its deflection limits. No scheduling of the gains with respect to the jammed position is necessary. For a jammed flap a single set of gains are adequate to restabilize the $X-33$ for jammed flap in a retrimmable
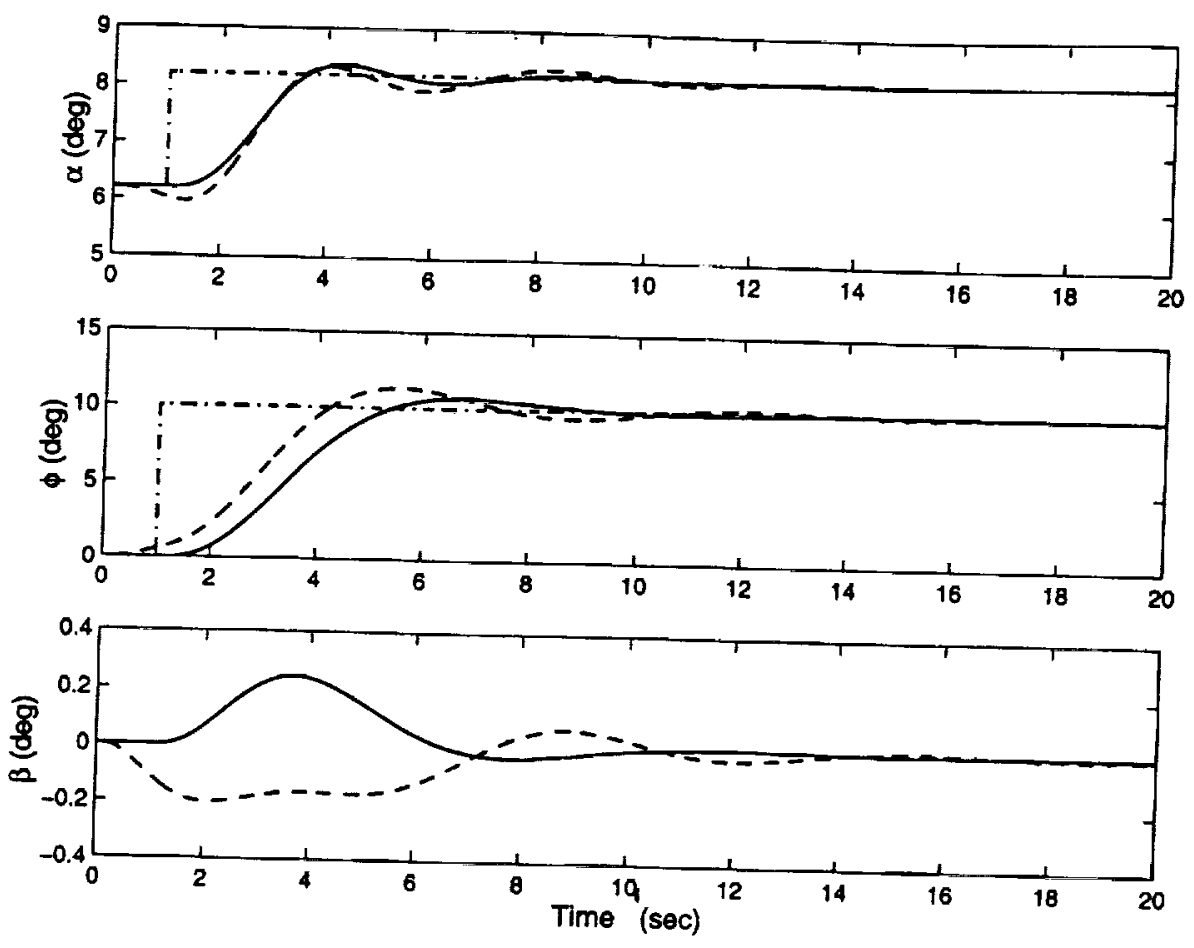

Fig. 3a Comparison of responses using PI-servo method with left inboard elevon runaway then jammed at -15 deg (-- ) and nominal controller
without a failure (-
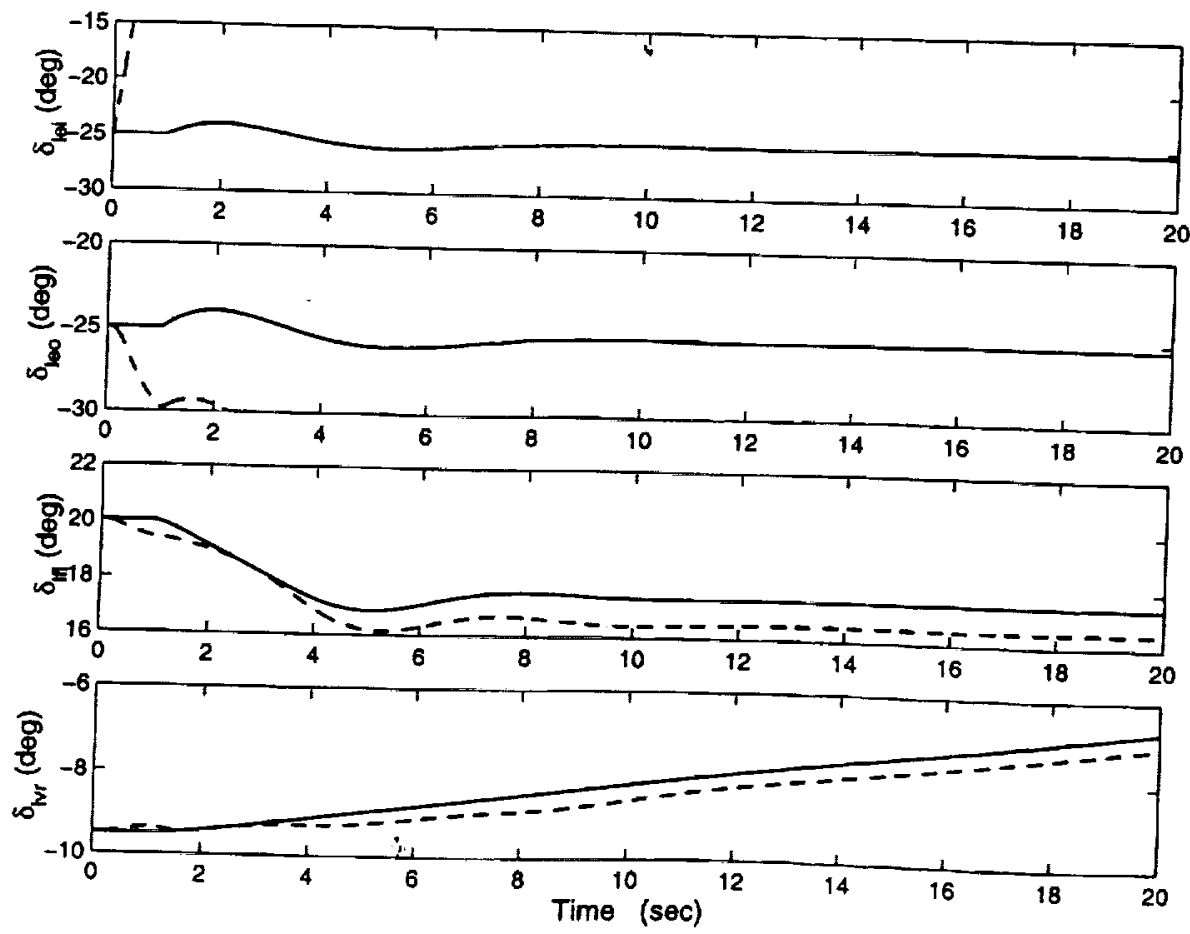

Fig. 3b Comparison of responses using PI-servo method with left inboard elevon runaway then jammed at -15 deg (--) and nominal controller 
position (cf., Sec. IV.B). This range cannot be increased by any gain scheduling with respect to the jammed flap position because this is the physical limit for the $\mathrm{X}-33$ to be able to retrim.

Figures $2 a-2 c$ show a time history comparison of the linearized model and the nonlinear six-degree-of-freedom simulator for the $X-33$ under the same control law. The results show a good comparison between the linear model and the six-degree-of-freedom simulation. No gain adjustments were made in this case and in the following cases when applying the control law to the nonlinear simulator. Even though these results matched, when using a linear model (linear plant and simple nonlinear actuators with rate limits and surface saturation limits) special attention must be taken when analyzing control effector activity or actuator response, especially for reconfiguration design. It is recommended that a high-fidelity nonlinear simulator be used to validate the linear design results. For the remaining position of the paper, the time-history results are from the nonlinear six-degree-of-freedom simulator.

Figures $3 a-3 c$ show how the servomechanism controller with a failure works compared to the nominal case without a failure. These figures in dashed line show the time histories of a runaway left inboard elevon failure that starts at $t=0$ and jams at $-15 \mathrm{deg}$. A longitudinal and lateral-directional guidance command tracking
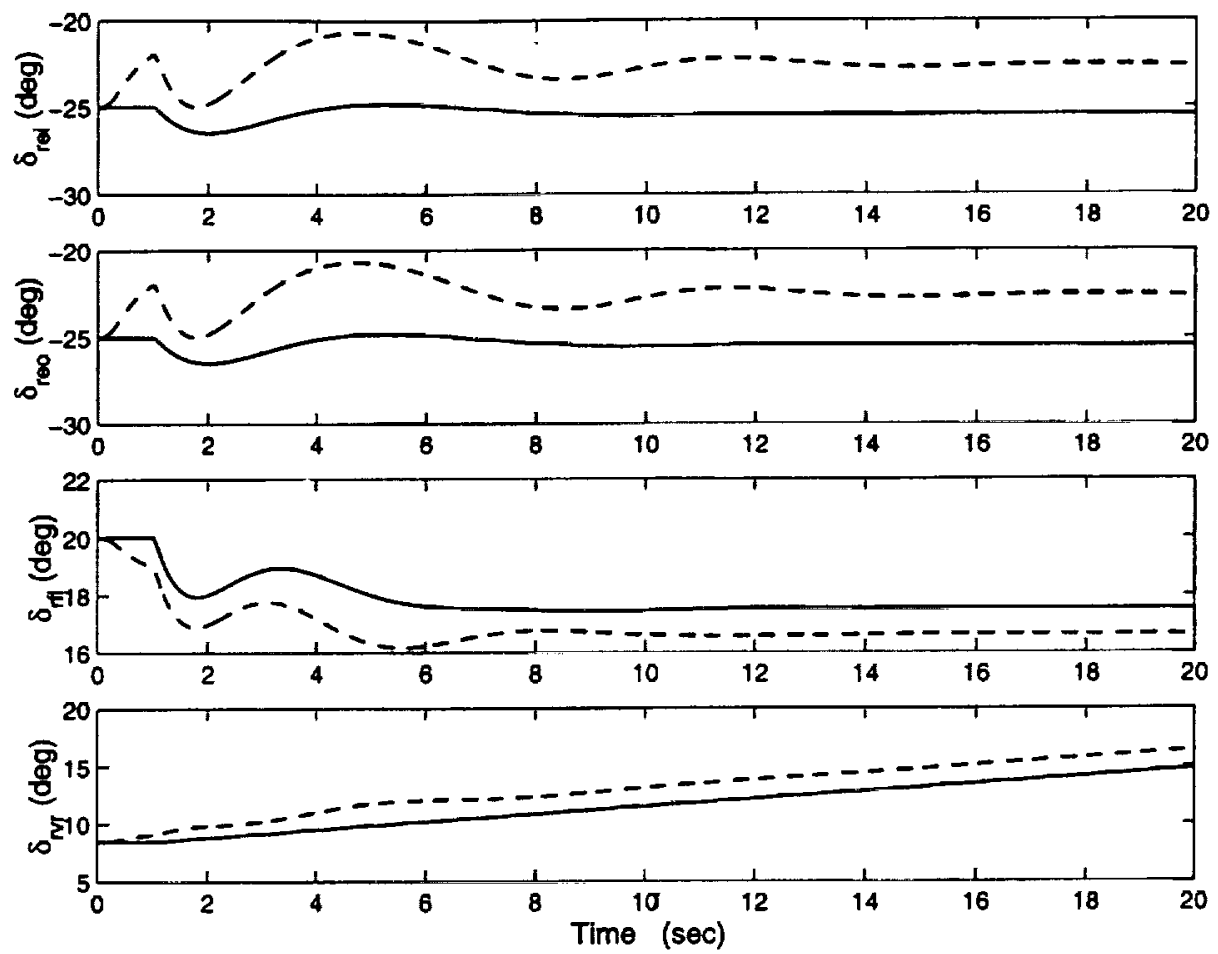

Fig. 3c Comparison of responses using PI-servo method with left inboard elevon runaway then jammed at -15 deg (---) and nominal controller without a failure $(\longrightarrow)$ for right-side control surfaces.
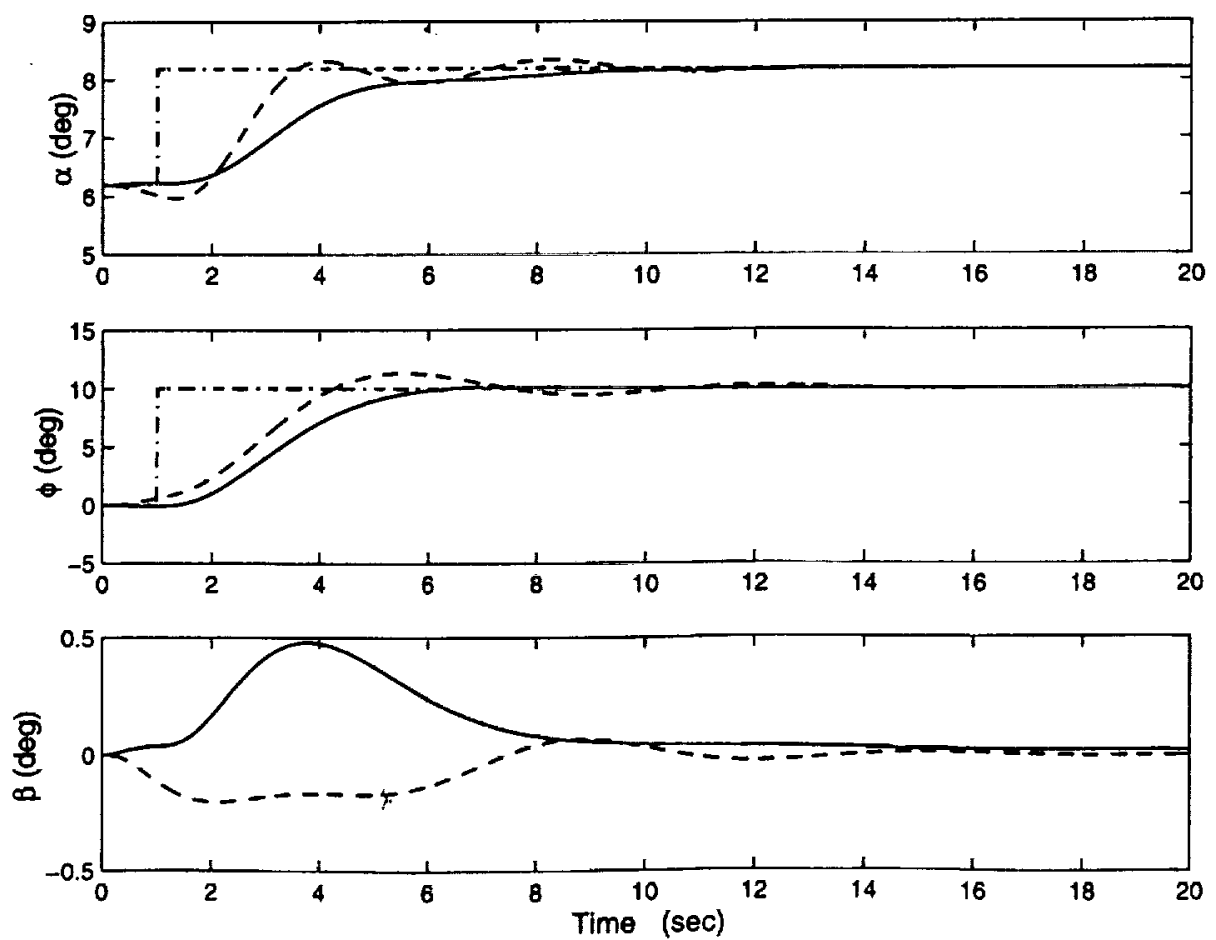

Fig. ta Comparison of responses using PI-servo method with left inboard elevon runaway then jammed at - 15 deg (---) and control-allocation method $(\longrightarrow)$. 
step input starts at $t=1$. The guidance commands are for simultaneous tracking of angle-of-attack and roll-angle commands during the failure because maneuvers in both axes are more demanding of the reconfigurable control system. Sideslip angle command remains at 0.0 deg for all test cases. Figure 3 al shows the commands and the resulting responses. The PI-servo controller tracks the commanded angle of attack of $8 \mathrm{deg}$ and the roll angle of 10 deg adequately. Figure $3 \mathrm{~b}$ shows the left surface positions, and Figure 3c shows the right surface positions. As can be seen in these figures, the servomechanism controller with a fuilure performs almost as well as the nominal controller without a failure.
This servomechanism control approach was found to work well for any janmed positions of any of the six aerosurfaces, excluding the flaps. For the jamming of one of the flaps not far from the trim position. the PI control system still performs well. Only when a thap is jamned at a position far from its trim position, the performance of the reconfigured PI control system begins to degrade considerably.

\section{Control-Allocation Reconfigurable Design}

A reference PI control law using all of the eight aerosurfaces of the healthy $X-33$ is designed using the servomechanism and LQR method to serve as the baseline control law
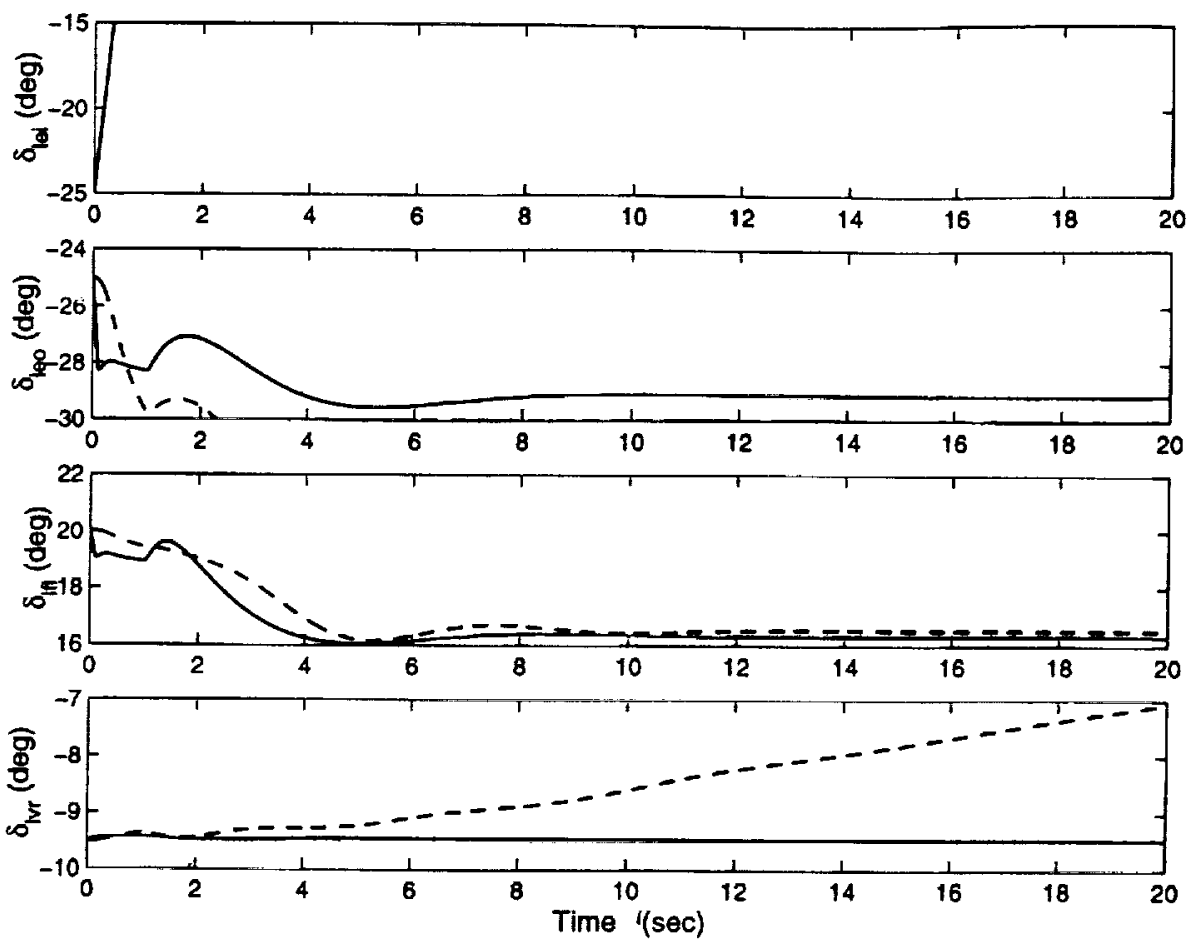

Fig. 4b Comparison of responses using PI-servo method with left inboard elevon runaway then jammed at -15 deg (-- ) and control-allocation method $(\longrightarrow)$ for left-side control surfaces.
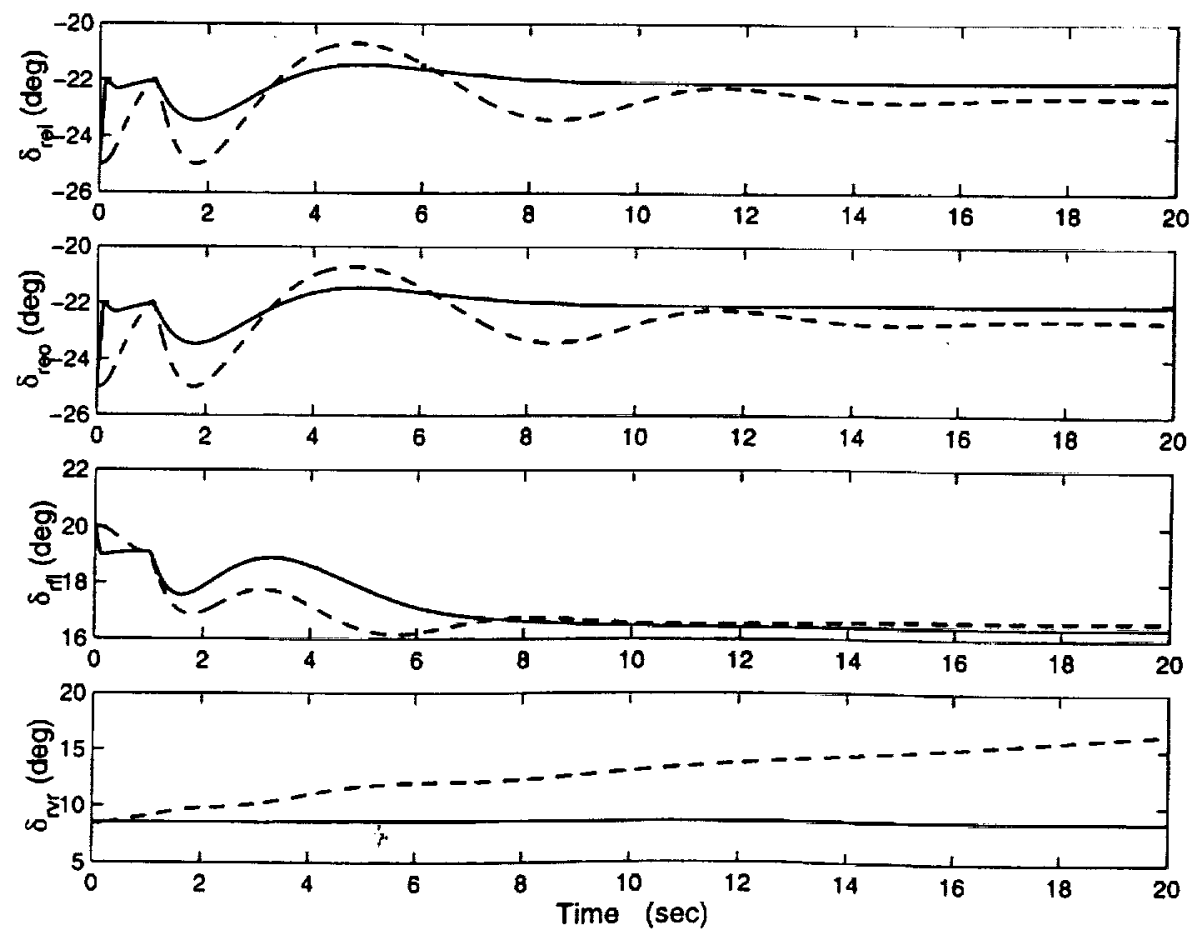

Fig. 4c Comparison of responses using PI-servo method with left inboard elevon runaway then jammed at -15 deg (---) and control-allocation method (—) for right-side control surfaces. 


$$
\begin{aligned}
& u^{*}=K_{i}^{*} x+k_{\phi}^{*} \int\left(\phi_{\mathrm{sm} l}-\phi\right) \mathrm{d} t+k_{\beta}^{*} \int\left(\beta_{\mathrm{s} m \mathrm{~m}}-\beta\right) \mathrm{d} t \\
& +k_{i}^{*} \int\left(\alpha_{c m i d}-\alpha\right) \mathrm{d} t
\end{aligned}
$$

The reference control law should not push the vehicle too hard for performance. Otherwise the vehicle after failure may not be able to generate the control actions the reference controller calls for. This woukl be the case when the set $(1$ in Ey. $(17)$ is emply, and if this happens too frequently, instability could occur.

The controlled output vector = used in Sec. III is chosen to be $z=(p r q \beta \alpha)^{T}=C_{z} x$. The $\beta$ and $\alpha$ are alded to $=$, in addition to
$P . r$, and $q$, because they are part of the comminded outputs of the $X$-33. But the difference in term of the response as compared to the case where $z=(p+q)^{T}$ was found to be incliscemible.

For each jammed surface the reference ${ }^{*}$ is always computed from $\mathrm{Eq}_{4}(37)$ at the current state $x$. The $Q_{1}$ and $Q_{2}$ matrices in Eq. (1.5) are chosen to be identity matrices, and $\varepsilon=0.000 .5$ is used. The control commands for the remaining seven aerosurfaces are obtained from the fixed-point iteration (27).

Figure 4 a shows the performance comparison of the controlallocation approach and the servo-PI controller for the case of a runaway left inboard that jams at -15 degs (see Fig. 3). Figures tb and $4 c$ depict the aerosurface variations for both methods. The results
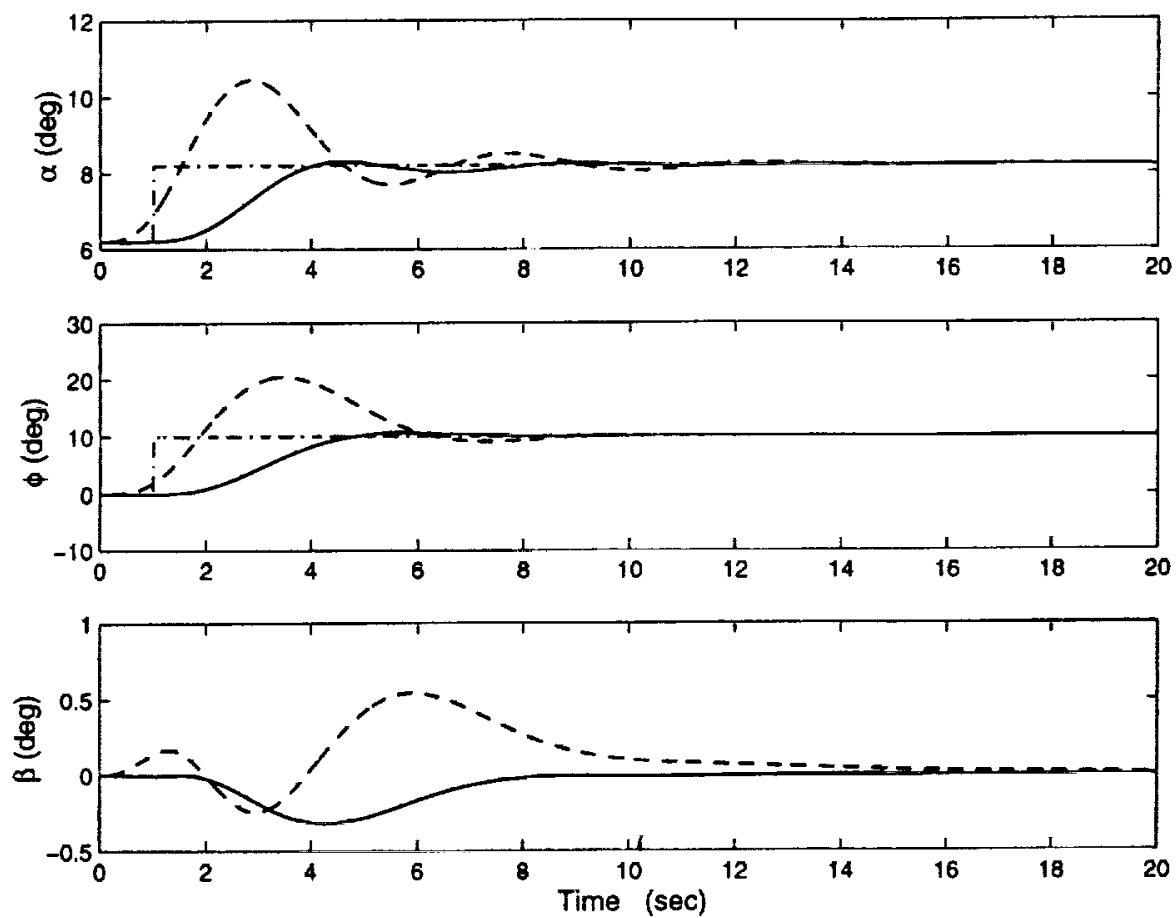

Fig. 5a Comparison of responses using Pl-servo method with right body flap runaway then jammed at 10 deg (---) and control-allocation method (-).
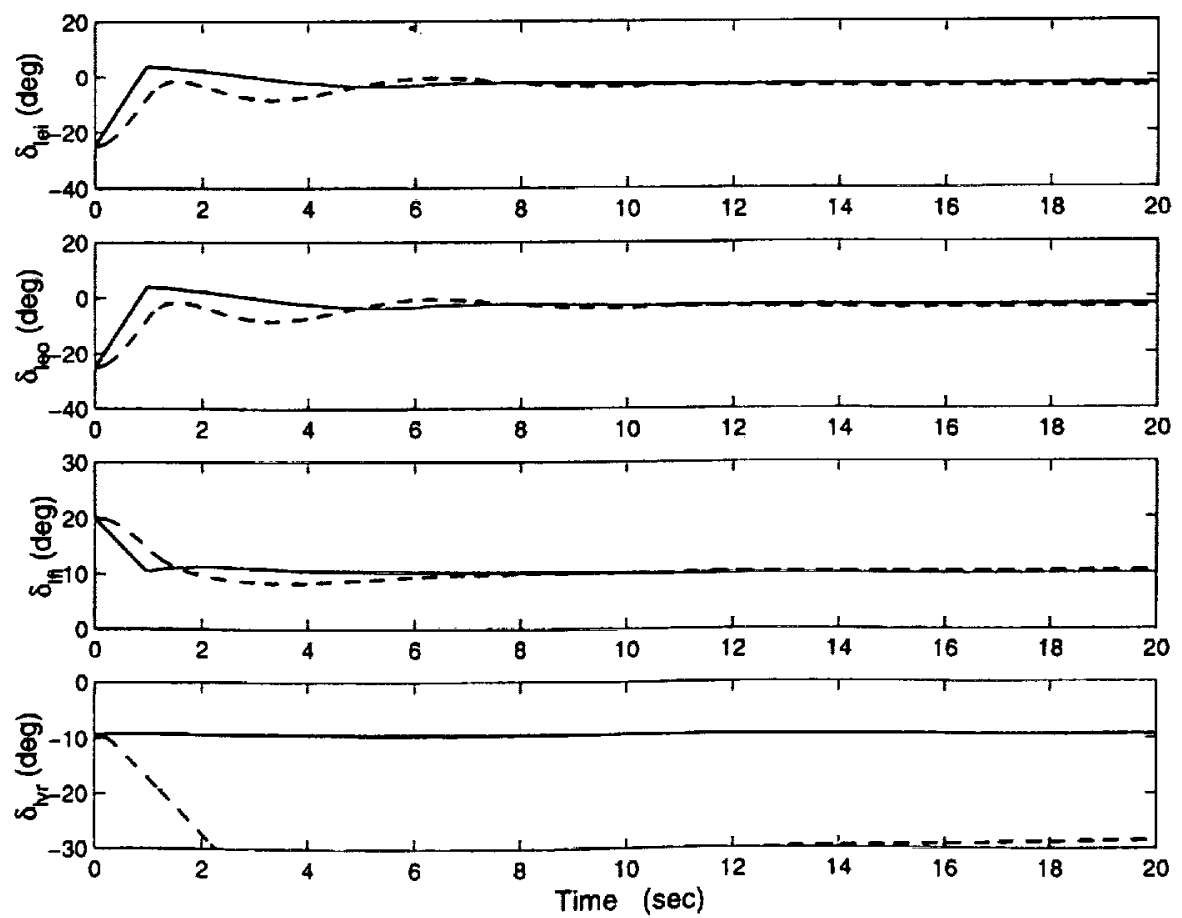

Fig. 5b Comparison of responses using PI-servo method with right body flap runaway then jammed at 10 deg (---) and control-allocation method $(-$ for left-side control surfaces. 

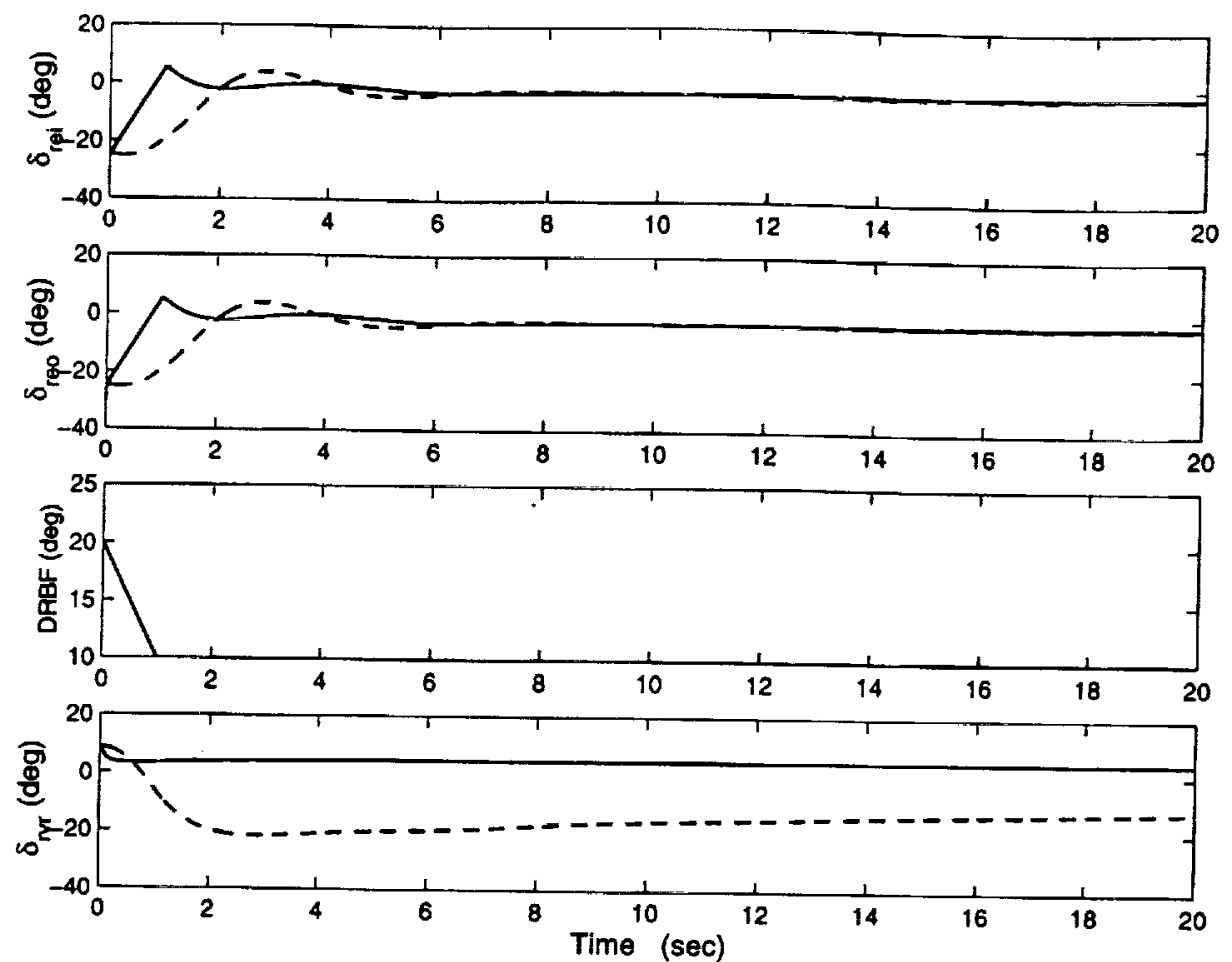
Fig. 5c Comparison of responses using PI-servo method with right body flap runaway then jammed at 10 deg (---) and control-allocation method
$(-)$ for right-side control surfaces.

show that the servo-PI controller has some overshoot, whereas the control-allocation method was well damped with low control surface activity (Figs. $4 \mathrm{~b}$ and $4 \mathrm{c}$ ).

Figure 5 a shows the performance comparison of the controlallocation approach and the servo PI controller for a runaway right body flap from 20 to $10 \mathrm{deg}$. Figures $5 \mathrm{~b}$ and $5 \mathrm{c}$ show the control surface positions for both methods. The control-allocation approach provided a good response in this and other similar difficult cases, whereas the servo-PI method did not have as good a performance. A tradeoff here is that the control-allocation method needs to know the surface and position that has failed, but the servo-PI method only needs to know what surface failed, not the position.

The responses seen in Figs. $4 \mathrm{a}$ and $5 \mathrm{a}$ under the control-allocation reconfiguration are similar. In fact, the control-allocation approach is found to provide uniformly good performance for all of the failure (jamming) cases where stabilization and command-tracking are possible with the remaining aerosurfaces. Especially in the challenging situations where a body flap is jammed, the control-allocation approach yields nearly the same good performance for any jammed position of a body flap within the retrimmable range. When the flap jammed position is outside the retrimmable range, instability occurs rapidly because some of the remaining operable surfaces become severely saturated when trying to counter the jammed flap.

\section{Conclusions}

Two methods for design of reconfigurable fight-control systems to handle jammed control surface are presented. One is based on a robust servomechanism design methodology. For the failure cases of jammed surface, the approach leads to a MIMO PI controller. The other method uses a QP-based control-allocation scheme to redistribute the operable control surfaces to cancel the influence of the jammed surface and still provide desired control moments and forces to the aircraft. The formulation is formally analyzed to justify its validity, and a globally convergent, simple fixed-point algorithm is developed for onboard implementation of the method. The servo-PI controllers are simple to implement, and the position of the failed surface need not be known. But a different controller is needed for each possible jammed surface. The control-allocition method on the other hand requires no separate designs for different jammed surfaces, but modest online computation and adeguate con- trol redundancy are needed. The control allocation does require the knowledge of the jammed position of the failed surface. Therefore the answer to the question of which design is more suitable depends on the onboard resources.

Applications of both approaches to reconfigurable entry flight control of the X-33 vehicle demonstrate the potential of the two methods. The servo-PI controller provides good performance in all but the most severe failure scenarios. In comparison, uniformly good and virtually the same performance is achieved by the controlallocation-based controller, regardless of which surface is jammed, as long as it is still possible to control the vehicle within the authority of the remaining surfaces.

Although only one jammed control surface is discussed throughout this work, it is straightforward to extend the two methods introduced here to the cases where jam of more than one surface occurs if the consideration of such an event is warranted.

Finally, we recognize that reconfigurable control design methods exist that can be applied to more general failure scenarios and are better suited to handle nonlinearity. ${ }^{1.4}$ But the methods discussed in this paper are consistent with the current framework of how flight control systems are designed and appear to be very effective for the restricted class of failures (jammed surfaces). A reconfigurable fight-control system designed with these methods can be easily evaluated and tested in accordance with today's well-established criteria and standards. This may prove to be important for the methods to gain acceptance in practice.

\section{Appendix: Linearized Model of the X-33}

The following is a linearized model of the X-33 near the terminal area energy management interface in entry flight. The flight conditions are Mach $=3.16$. height $=97,167 \mathrm{ft}, \alpha_{\text {trim }}=6.23 \mathrm{deg}$. $\theta_{\text {trim }}=0.922 \mathrm{deg}, \phi_{\mathrm{trim}}=\beta_{\mathrm{tnm}}=0$, and weight $=78,593 \mathrm{lbm}$. Let $u=\left(\delta_{\mathrm{rel}} \delta_{\mathrm{lei}} \delta_{\mathrm{rrl}} \delta_{\mathrm{lH}} \delta_{\mathrm{rvr}} \delta_{1 \mathrm{rr}} \delta_{\mathrm{rew}} \delta_{\mathrm{leo}}\right)^{T}$ be the control surface perturbations from the trim values, where $\delta_{\mathrm{ret}} . \delta_{\mathrm{let}}$ are the right and left inboard elevons, $\delta_{\mathrm{rt}}$. $\delta_{\mathrm{lt}}$ the right and left body flaps, $\delta_{\mathrm{rvr}}, \delta_{\mathrm{lvr}}$ the right and left rudders, and $\delta_{\text {reo }} . \delta_{\text {leo }}$ the right and left outboard elevons

All of the control-surface deflections are in degrees. The sign convention is positive body flap deflection is down, positive elevon deflection is down, and positive rudder delection is left looking forward. The surface trim values are $19.98 \mathrm{deg}$ for the body flaps, 
-24.88 deg for the inbourd and outboard elevons, and $9.4 \mathrm{deg}$ for the right rudder and $-9.43 \mathrm{deg}$ for the left rudder. Let the perturbations from the trim conditions be $x=(p r \beta \phi \psi \alpha q \theta v)^{T}$, where the components are, in the order of appearance in $x$, roll rate, yaw rate, sideslip angle, roll angle, yow angle, angle of attack, pitch rate, pitch angle, and forward velocity. The units are in degrees/second for angular rates, degrees for angles, and feet/second for velocity. The linearized dynamics of the $\mathrm{X}-33$ at the preceding flight conditions are given by

$$
\dot{x}=A x+B u
$$

where

$$
A=\left[\begin{array}{cc}
A_{\text {lat }} & 0 \\
0 & A_{\text {lon }}
\end{array}\right]
$$

with

$$
\begin{aligned}
& A_{\text {lat }}=\left[\begin{array}{ccccc}
-0.099170 & 0.020570 & 2.805001 & 0.000001 & 0.000001 \\
-0.002640 & -0.031920 & -0.674200 & 0 & 0 \\
-0.108500 & -0.994000 & -0.029750 & 0.010210 & 0.000034 \\
1.0 & 0.016090 & -0.000148 & 0.000016 & 0 \\
0 & 1.0 & 0.000120 & -0.000013 & 0.000122
\end{array}\right] \\
& A_{\text {lon }}=\left[\begin{array}{cccc}
-0.064190 & 1.000001 & 0.000884 & -0.000130 \\
-1.424000 & -0.051730 & 0.000001 & 0 \\
-.0000138 & 1.0 & 0.000014 & 0.000003 \\
-0.550041 & 0 & -0.559090 & -0.013375
\end{array}\right]
\end{aligned}
$$

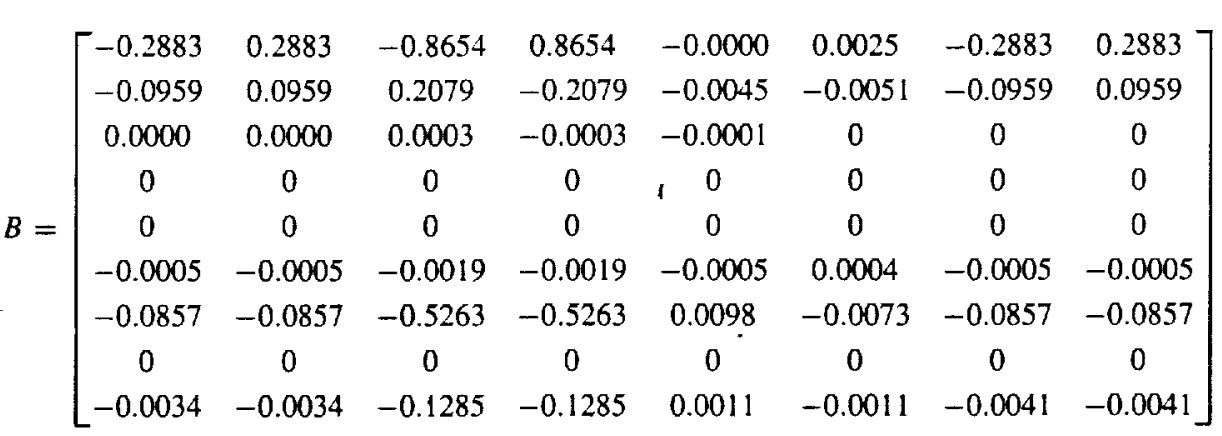

'Brinker. J. S., and Wise, K. A., "Reconfigurable Flight Controls for a Tailless Advanced Fighter Aircraft," Proceedings of Guidance, Novigation. and Cuntrol Conference, Vol, I, AIAA, Reston, VA. 1998. pp. 75-87.

'Wagner, E., Burken, J., Hanson. C., and Wohletz., J., "Deterministic Reconfigurable Control Design for the X-33 Vehicle." Proceedings of Guidance. Navigation. and Comtrol Conference, Vol. 3, AIAA, Reston, VA, 1998, pp. $|342-135|$

${ }^{3}$ Morse, W. D., and Ossman, K. A., "Model Following Reconfigurable Flight Control System for the AFTI/F-16," Journal of Cuidance, Control, and Dynamics, Vol. 13, No. 6, 1990, pp. 969-976.

${ }^{4}$ Calise, A. J., Lee, S., and Sharma, M.. "Direct Adaptive Reconfigurable Control of a Tailless Fighter Aircraft," Proceedings of Guidunce, Navigation. and Control Conference, Vol. I, AIAA, Reston, VA, 1998, pp. 88-97.

${ }^{5}$ Desoer, C. A., and Wang, Y. T., "Linear Time-Invariant Robust Servomechanism Problem: A Self-Contained Exposition," Control and Dynamic Sistems, edited by C. T. Leondes, Vol. 16, Academic, New York, 1980. pp. 81-129.

${ }^{6}$ Davison, E. J., "The Robust Control of a Servomechanism Problem for
Note that the $A$ matrix is decoupled in the longitudinal and lateral directions, but the $B$ matrix is not.

\section{Acknowledgment}

The authors at Iowa State University gratefully acknowledge the support for this research by the NASA Dryden Flight Research Center under Corporative Agreement NCC4-117.
Linear Time-Invariant Multivariable Systems," IEEE Transactions on Automatic Control, Vol. AC-21, No. I, 1976. pp. 25-34.

'Enns, D., "Control Allocation Approaches," Proceedings of Guidance, Navigation, and Control Conference, Vol. I, AIAA, Reston, VA, 1998, pp. 98-108.

${ }^{8}$ Luenburger, D. G., Linear and NonlinearProgramming, 2nd ed., Addison Wesley Longman, Reading, MA, 1984, pp. 425, 426.

${ }^{9}$ Lu. P., "Constrained Tracking Control of Nonlinear Systems," Systems and Control Letters, Vol. 27, 1996, pp. 305-314. 
Public reporting burden for this collection of information is estimated to average 1 hour per response, including the time for reviewing instructions, searching existing data sources, gathering and maintaining the data needed, and completing and reviewing the collection of information. Send comments regarding this burden estimate or any other aspect of this collection of information,

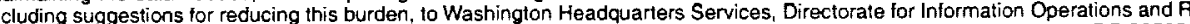
VA 22202-4302, and to the Office of Management and Budget, Paperwork Reduction Project (0704-0188), Washington, DC 20503.

\begin{tabular}{|l|l|l|l|}
\hline 1. AGENCY USE ONLY (Leave blank) & $\begin{array}{l}\text { 2. REPORT DATE } \\
\text { June } 2001\end{array}$ & $\begin{array}{l}\text { 3. REPORT TYPE AND DATES COVERED } \\
\text { Journal Article }\end{array}$ \\
\hline
\end{tabular}

4. TITLE AND SUBTITLE

Two Reconfigurable Flight-Control Design Methods: Robust

Servomechanism and Control Allocation

6. AUTHOR(S)

John J. Burken, Ping Lu, Zhenglu Wu, and Cathy Bahm

7. PERFORMING ORGANIZATION NAME(S) AND ADDRESS(ES)

8. PERFORMING ORGANIZATION

REPORT NUMBER

NASA Dryden Flight Research Center

P.O. Box 273

H-2433

Edwards, California 93523-0273

WU 242-33-02-00-23-00-TA5

9. SPONSORING/MONITORING AGENCY NAME(S) AND ADDRESS(ES)

10. SPONSORING/MONITORING

AGENCY REPORT NUMBER

National Aeronautics and Space Administration

Washington, DC 20546-0001

11. SUPPLEMENTARY NOTES

Presented at AIAA Guidance Navigation and Control Conference, Portland, Oregon, August 9-11, 1999, AIAA-99-4134.

12a. DISTRIBUTION/AVAILABILTTY STATEMENT

12b. DISTRIBUTION CODE

Unclassified-Unlimited

Subject Category 08

13. ABSTRACT (Maximum 200 words)

Two methods for control system reconfiguration have been investigated. The first method is a robust servomechanism control approach (optimal tracking problem) that is a generalization of the classical proportional-plus-integral control to multiple input-multiple output systems. The second method is a controlallocation approach based on a quadratic programming formulation. A globally convergent fixed-point iteration algorithm has been developed to make onboard implementation of this method feasible. These methods have been applied to reconfigurable entry flight control design for the X-33 vehicle. Examples presented demonstrate simultaneous tracking of angle-of-attack and roll angle commands during failures of the right body flap actuator. Although simulations demonstrate success of the first method in most cases, the control-allocation method appears to provide uniformly better performance in all cases.

14. SUBJECT TERMS

Failure control system design, Reconfigurable flight controls, Robust servomechanism design

\begin{tabular}{|l|l|l|}
\hline 17. SECURITY CLASSIFICATION & $\begin{array}{l}\text { 18. SECURITY CLASSIFICATION } \\
\text { OF REPORT }\end{array}$ & $\begin{array}{l}\text { 19. SECURITY CLASSIFICATION } \\
\text { OF ABIS PAGE } \\
\text { Unclassified }\end{array}$ \\
Unclassified & Unclassified \\
\hline
\end{tabular}

